\title{
Continuous constitutive model for bimodulus materials with meshless approach
}

\author{
T. Huang ${ }^{1}$, Q.X. Pan $^{1}$, J. Jin ${ }^{1}$, J.L. Zheng ${ }^{1}$, P.H. Wen ${ }^{2 *}$ \\ ${ }^{1}$ School of Traffic and Transportation Engineering, Changsha University of Technology and Science, China \\ ${ }^{2}$ School of Engineering and Materials Science, Queen Mary, University of London, London E1 4NS, UK
}

\begin{abstract}
One of the difficulties in dealing with bimodular materials including composites, rock and asphalt-mixture material is the discontinuity of Young's modulus when the principal stress changes sign, i.e. from a tensile stress state to a compressive stress state. According to the general elastic theory proposed by Ambartsumyan, there are two kinds of domains in which the coefficients of elasticity are constant. The discontinuity of Young's modulus causes divergence in the computational procedure. In order to overcome this difficulty, two continuous modes for bimodular materials are proposed in this paper. The nonlinear equilibrium equations have been formulated with a continuous constitute equation of stress and strain. The meshless finite block method is successful in solving the nonlinear problems for bimodular materials. The numerical solutions of the meshless finite block method in a strong form are obtained using an iterative technique. The degree of accuracy and convergence of the proposed technique is demonstrated by directly comparing the achieved results with the finite element method and analytical solutions.
\end{abstract}

Key words: Nonlinear constitution equation, bimodular material, composites, meshless finite block method, mapping technique, differential matrix.

*Corresponding: p.h.wen@qmul.ac.uk (P.H.Wen) 


\section{Introduction}

In classical elasticity theory, it is well known that a material has the same elastic properties in tension and compression. However, this is only a simplified modeling and does not account for material non-linearity. In material science and engineering structures, it is shown that the strength of some materials such as composites, poroelasticity, rocks, concrete and asphaltmixture differ significantly during tensile and compressive loading. Furthermore, mechanical properties such as the elastic modulus and the Poisson's ratio of the listed materials can vary under tensile and compressive loading. Thus, these materials are defined as bimodular materials which have a different Young's modulus, shear modulus and Poisson's ratio in respect to tensile and compression loading. In such cases, there are no explicit expressions related to the Young's modulus, the Poisson's ratio and the shear modulus [1-3]. Experimental data shows that the constitutive relationship between stress and strain has different slopes as shown in Figure 1(a). There is also a jump in the Young's modulus when the stress changes direction as shown in Figure 1(b). It has been observed that most materials, including ceramics and some composites, have different tensile or compressive strain when subjected to the same tensile or compressive stress.

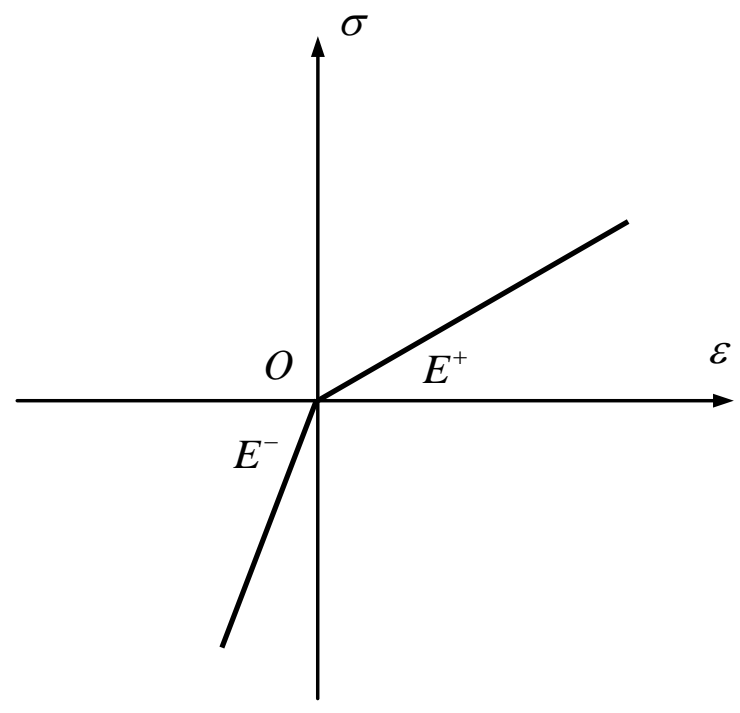

(a)

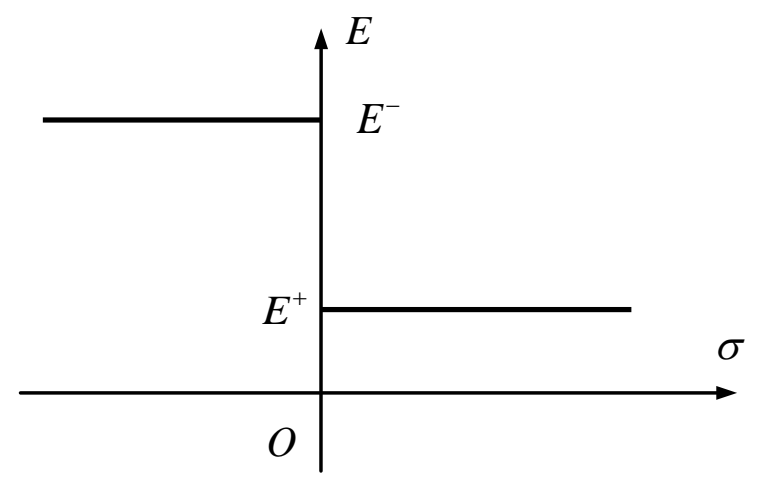

(b)

Figure 1. Relationship of stress and strain from experimental study: (a) constitution of bimodules material; (b) discontinuity of Young's modulus. 
Nowadays, two material models are widely used in both theoretical analysis and in engineering application for bimodular materials investigation. The first one is the criterion of positive-negative signs in the longitudinal strain of fibers, proposed by Bert [4]. This model is applicable to orthotropic materials and widely used in laminated composites [5-10]. The second model is the criterion of positive-negative signs of principal stress, proposed by Ambartsumyan [11,12]. This model is applicable to isotropic materials. Ambartsumyan and Khachatryan [13] investigated the constitutive relations of bimodular materials theoretically and derived some analytical solutions for two and three dimensional problems, including a bar with gravity, beam and rectangular flat plate under bending. In the criterion of positive-negative signs of principal stress, the stress state in a principal direction is a key issue in the analysis. It is considered that Young's modulus depends not only on the material properties, but also on the stress state at collocation point in the domain.

Due to material non-linearity, the numerical simulation techniques adopted by the Finite Element Method (FEM) is based on an iterative strategy. However, commercial finite element analysis software such as ANSYS, NASTRAN, ABAQUS and DYNA3D have no functions to deal with bimodular problems. Therefore, the development of finite element analysis for bimodular materials has become the focus of many researchers and engineers $[14,15,16]$. Since there is a discontinuity of Young's modulus at the origin shown in Figure 1(b), two kinds of a domain are specified in the domain. Yang and Wang [17] proposed a continuous model to treat this discontinuity. The analytical solutions are derived for beam and plate bending problems $[18,19,20]$.

The development of new methods and skill in computational mechanics is always an attractive proposition in regards to solving difficult engineering problems. Mesh free ideas date back to 1977 with Monaghan and Gingold [21] and Lucy [22] developing a Lagrangian method based on the Kernel Estimates method for modelling problems in astrophysics. Meshless approximation was proposed by Nayroles et al [23] for the diffuse equation and later, elementfree Galerkin method and re-producing kernel particle methods by Belyschko et al [24] and Liu et al [25]. Recently, Atluri and his colleagues [26-30] developed the Meshless Petrov-Galerkin methods (MLPGs) using the moving least-square (MLS) approximation which provide a rational basis for constructing meshless methods. The Local Boundary Integral Equation method (LBIE) with either moving least square or radial basis function (RBF) has been developed by 
Sladek et al [31,32,33] for the boundary value problems in anisotropic non-homogeneous media. A comprehensive review of meshless methods (MLPG) can be found in the book [30] by Atluri. Based on the point collocation method, the meshless Finite Block Method (FBM) was first proposed to solve elasticity problems by Wen and Cao [34]. Later, the FBM was successfully applied to the study of non-linear elasticity including heat conduction, contact and fracture mechanics by Li and Wen [35] and Li et al [36,37]. The unique feature of the FBM is that the domain is discretized into several blocks and the partial differential matrices are applied for each block with the mapping technique. In this paper, two continuous models in the constitution equation for bimodular materials are proposed in order to improve the computational accuracy and convergence with an iterative algorithm by using the FBM. In the principal coordinates, the Hook's law in elasticity matrix related to the principal stresses and strains is formulated for two and three dimensional problems. The stress-Young's modulus relationship is interpolated by a linear or a hyperbolic tangent function. A set of algebraic equation from the partial differential equations in a strong form is formulated in terms of the nodal values of displacement. Due to the non-linearity of material property, an iterative method is applied in the computation.

In this paper, the meshless FBM method is applied to high non-linear elasticity problems in engineering and the results are compared against the equivalent FEM results. The FEM method is widely accepted as the benchmark for numerical analysis across industry and among researchers. The accuracy and divergence of the method are observed and comparisons with analytical solution and FEM have been made for beam bending, rectangular plate with a circular hole and hollow/solid cylinders under compressive load.

\section{Bimodular material governing equations}

Two linear stress-strain relationships with different slopes under either tension or compression loadings are shown in Figure 1(a). The analytical theory proposed by Ambartsumyan [11] can be summarized below with two kinds of domain.

\subsection{General elasticity law}

From the assumption of elasticity in the principal directions and the linear Hook's law gives

$$
\varepsilon_{\alpha}=a_{11} \sigma_{\alpha}+a_{12} \sigma_{\beta}+a_{13} \sigma_{\gamma},
$$




$$
\begin{aligned}
& \varepsilon_{\beta}=a_{12} \sigma_{\alpha}+a_{22} \sigma_{\beta}+a_{23} \sigma_{\gamma}, \\
& \varepsilon_{\gamma}=a_{13} \sigma_{\alpha}+a_{23} \sigma_{\beta}+a_{33} \sigma_{\gamma}, \\
& \gamma_{\alpha \beta}=\gamma_{\beta \gamma}=\gamma_{\alpha \gamma}=0,
\end{aligned}
$$

where the coefficients $a_{i j}$ depend on the stress state as

(1) If $\sigma_{\alpha}>\sigma_{\beta}>\sigma_{\gamma}>0$, we have

$a_{11}=a_{22}=a_{33}=\frac{1}{E^{+}} ; a_{12}=a_{13}=a_{23}=-\frac{v^{+}}{E^{+}}$

(2) If $\sigma_{\gamma}<\sigma_{\beta}<\sigma_{\alpha}<0$, we have

$a_{11}=a_{22}=a_{33}=\frac{1}{E^{-}} ; a_{12}=a_{13}=a_{23}=-\frac{v^{-}}{E^{-}}$

(3) If $\sigma_{\alpha}>\sigma_{\gamma}>0$ and $\sigma_{\beta}<0$, we have

$a_{11}=a_{33}=\frac{1}{E^{+}} ; a_{22}=\frac{1}{E^{-}} ; a_{12}=a_{13}=a_{23}=-\frac{v^{+}}{E^{+}}=-\frac{v^{-}}{E^{-}}$

(4) If $\sigma_{\beta}>0$ and $\sigma_{\gamma}<\sigma_{\alpha}<0$, we have

$a_{22}=\frac{1}{E^{+}} ; a_{11}=a_{33}=\frac{1}{E^{-}} ; a_{12}=a_{13}=a_{23}=-\frac{v^{+}}{E^{+}}=-\frac{v^{-}}{E^{-}}$

Cases (1) and (2) are defined as the first kind, cases (3) and (4) are the second kind. For the first kind, the classic linear elastic theory is valid. In the Cartesian's coordinate system, the principal direction along the principal stresses $\sigma_{\alpha}, \sigma_{\beta}$ and $\sigma_{\gamma}$ are defined as shown in Figure 2 as $\alpha=\left(l_{1}, l_{2}, l_{3}\right), \beta=\left(m_{1}, m_{2}, m_{3}\right), \gamma=\left(n_{1}, n_{2}, n_{3}\right)$. Then, the strain and stress analysis gives [11]

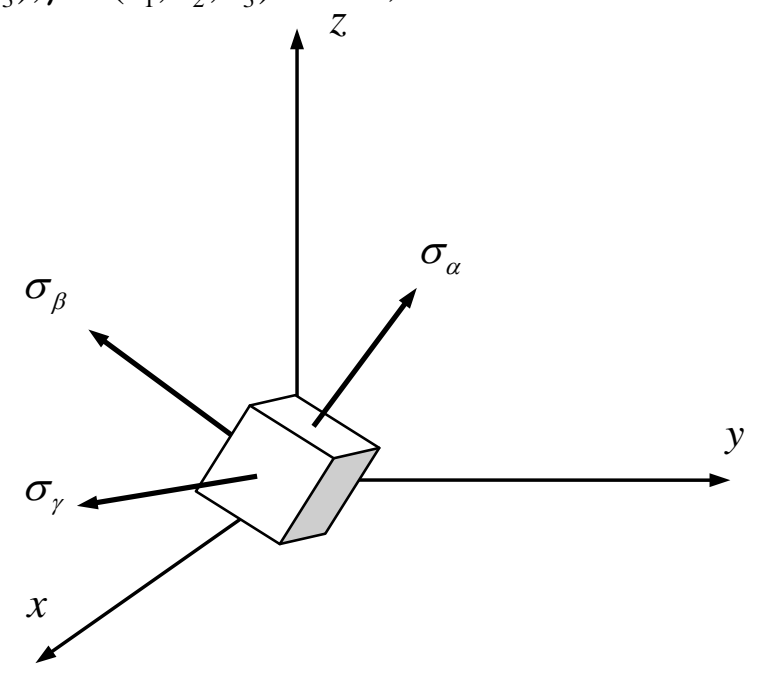

Figure 2. Principal stresses and their directions in Cartesian's coordinate system. 


$$
\begin{aligned}
& \varepsilon_{x}=a_{11} \sigma_{x}+a_{12}\left(\sigma_{y}+\sigma_{z}\right)+B_{3} m_{1}^{2} \sigma_{\beta}+B_{2} n_{1}^{2} \sigma_{\gamma}, \\
& \varepsilon_{y}=a_{11} \sigma_{y}+a_{12}\left(\sigma_{z}+\sigma_{x}\right)+B_{3} m_{2}^{2} \sigma_{\beta}+B_{2} n_{2}^{2} \sigma_{\gamma}, \\
& \varepsilon_{z}=a_{11} \sigma_{z}+a_{12}\left(\sigma_{x}+\sigma_{y}\right)+B_{3} m_{3}^{2} \sigma_{\beta}+B_{2} n_{3}^{2} \sigma_{\gamma}, \\
& \gamma_{x y}=2 A_{1} \tau_{x y}+2 B_{3} m_{1} m_{2} \sigma_{\beta}+2 B_{2} n_{1} n_{2} \sigma_{\gamma}, \\
& \gamma_{y z}=2 A_{1} \tau_{y z}+2 B_{3} m_{2} m_{3} \sigma_{\beta}+2 B_{2} n_{2} n_{3} \sigma_{\gamma}, \\
& \gamma_{x z}=2 A_{1} \tau_{x z}+2 B_{3} m_{1} m_{3} \sigma_{\beta}+2 B_{2} n_{1} n_{3} \sigma_{\gamma},
\end{aligned}
$$

where

$$
\begin{aligned}
& \varepsilon_{x}=\frac{\partial u}{\partial x}, \varepsilon_{y}=\frac{\partial u}{\partial y}, \varepsilon_{z}=\frac{\partial w}{\partial z}, \\
& \gamma_{x y}=\frac{\partial u}{\partial y}+\frac{\partial v}{\partial x}, \gamma_{y z}=\frac{\partial v}{\partial z}+\frac{\partial w}{\partial y}, \gamma_{x z}=\frac{\partial w}{\partial x}+\frac{\partial u}{\partial z} \\
& A_{1}=a_{11}-a_{12}, B_{2}=a_{33}-a_{11}, B_{3}=a_{22}-a_{11},
\end{aligned}
$$

in which $u, v, w$ are displacements and $A$ and $B$ are coefficients. Consider the second kind only here, i.e. $B_{2}=0$, and (6) and (7) give

$$
\begin{aligned}
\sigma_{x} & =2 A \varepsilon_{x}+B e-B_{3}\left(B+2 A m_{1}^{2}\right) \sigma_{\beta}, \\
\sigma_{y} & =2 A \varepsilon_{y}+B e-B_{3}\left(B+2 A m_{2}^{2}\right) \sigma_{\beta}, \\
\sigma_{z} & =2 A \varepsilon_{z}+B e-B_{3}\left(B+2 A m_{3}^{2}\right) \sigma_{\beta}, \\
\tau_{x y} & =A\left(\gamma_{x y}-2 B_{3} m_{1} m_{2} \sigma_{\beta}\right), \\
\tau_{y z} & =A\left(\gamma_{y z}-2 B_{3} m_{2} m_{3} \sigma_{\beta}\right), \\
\tau_{x z} & =A\left(\gamma_{x z}-2 B_{3} m_{1} m_{3} \sigma_{\beta}\right),
\end{aligned}
$$

where

$$
A=\frac{1}{2 A_{1}}, B=-\frac{a_{12}}{A_{1}\left(a_{11}+2 a_{12}\right)}, e=\varepsilon_{x}+\varepsilon_{y}+\varepsilon_{z}=\varepsilon_{\alpha}+\varepsilon_{\beta}+\varepsilon_{\gamma} .
$$

\subsection{Continuous modelling of Young's modulus}

It is apparent there are two types of domain determined by the stress state from (1) to (10). All coefficients in the general elasticity law are dependent on the stress state in the field, which constructs the nonlinearity. Apart from that, there is also a jump between the different types of domain and this is the main cause of the numerical computational divergence. 
Heaviside variation (bimodules shown in Figure 3): the discontinuity of the Young's modulus by Ambartsumyan can be expressed as

$$
E(\sigma)=E^{-}+\left(E^{+}-E^{-}\right) H(\sigma)
$$

where $H(\sigma)$ is the Heaviside function. In order to establish a continuous model of Young's modulus, a linear and smooth modulus model is proposed as shown in Figure 3. Clearly there are no sub-domains of the stress state in the formulations.

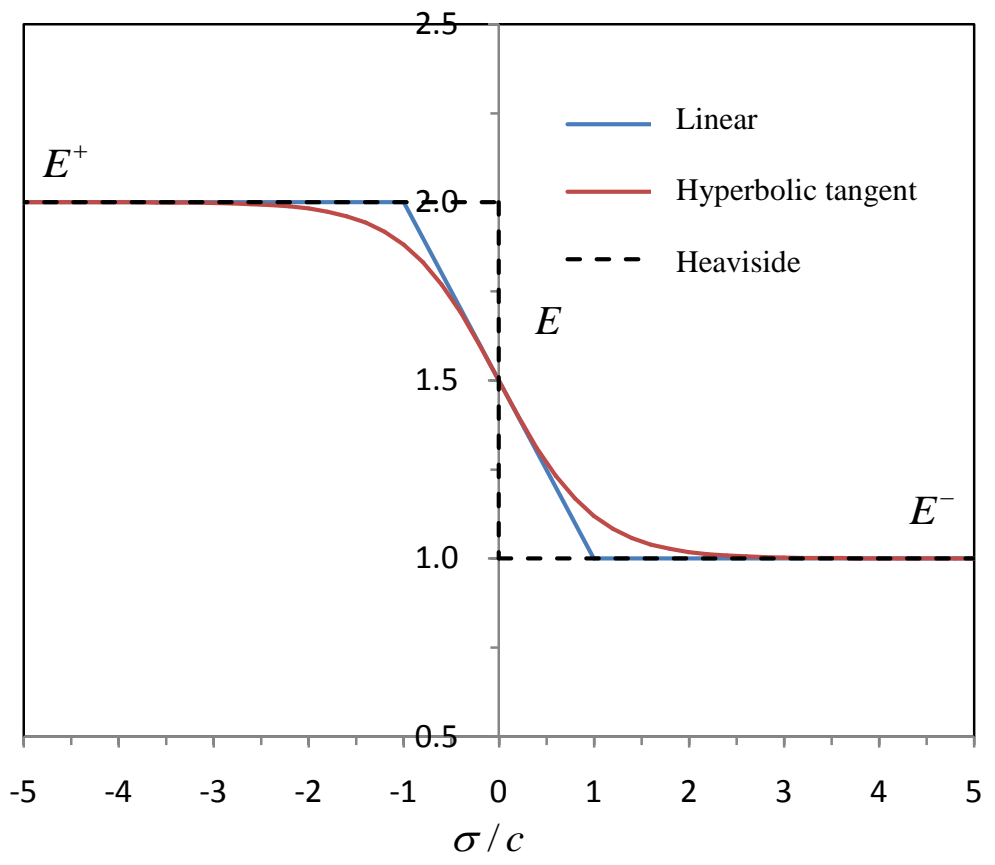

Figure 3. Continuous models of Young's modulus.

Linear variation: the Young's modulus is represented as

$$
E(\sigma)=\left\{\begin{array}{cc}
\frac{E^{+}+E^{-}}{2}+\frac{E^{+}}{2 c} \sigma & |\sigma| \leq c \\
E^{-} & \sigma<-c
\end{array}\right.
$$

where $c$ is the characteristic stress to be determined by the curve fitting technique with experimental data under uniaxial tensile/compressive loads. This parameter is normalized using the applied load $c=\beta \sigma_{0}$, where $\beta$ is the character dimensionless factor and $\sigma_{0}$ as utilised in this paper denotes the applied pressure load. 
Hyperbolic tangent variation: the variation of the Young's modulus is smooth and can be written as

$$
E(\sigma)=\frac{E^{+}+E^{-}}{2}+\frac{E^{+}-E^{-}}{2} \tanh (\sigma / c)
$$

where $\tanh (\sigma / c)$ is a hyperbolic function. The comparison of these two continuous modes is illustrated in Figure 3. Apparently, for a large absolute value of stress, the Young's modulus is taken either as $E^{+}$or $E^{-}$for both continuous modes. Figure 4 shows the relation between the strain and the normalized stress. The difference between the bimodules model and the continuous model can be seen clearly. The linear model is much closer to the bimodules mode than the hyperbolic tangent model. However, the hyperbolic model is a smooth model and is therefore, easier to formulize all partial differential equations in a mathematical model. In addition, the numerical solutions show that the difference between the linear and the hyperbolic modes are too small.

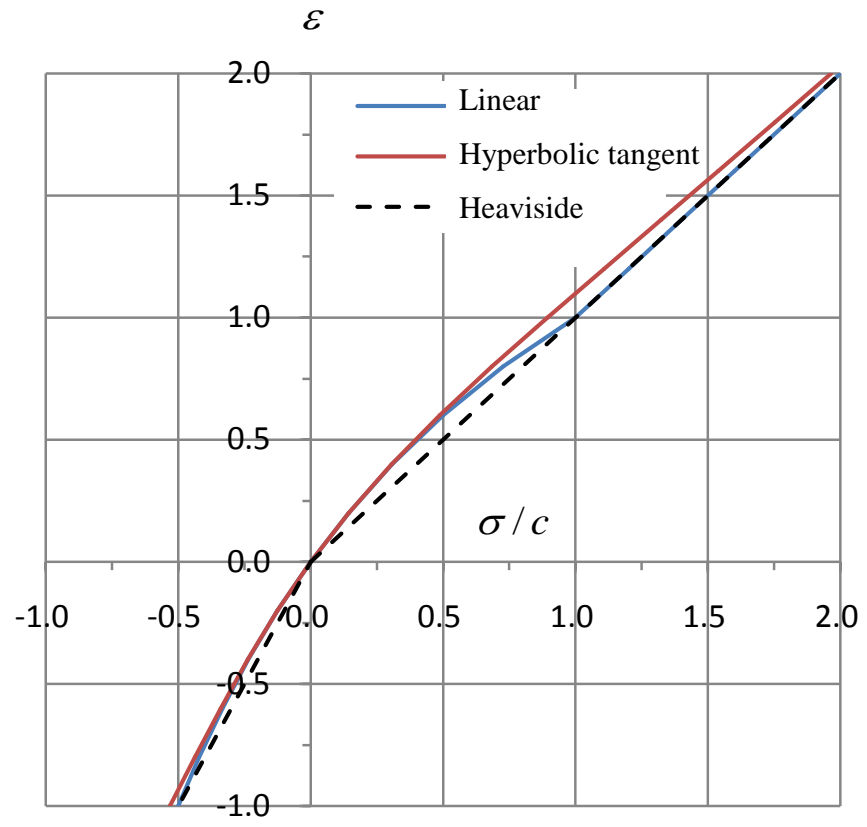

Figure 4. Constitute relationship between stress and strain in one dimensional problem. 


\section{Formulations for bimodular material}

By introducing the continuous mode, we do not need to divide the field into two types in order to specify all the coefficients of the material in the principal stress directions. All coefficients in (1) can be written as

$$
\begin{aligned}
& a_{11}=\frac{1}{E\left(\sigma_{\alpha}\right)} ; a_{22}=\frac{1}{E\left(\sigma_{\beta}\right)} ; a_{33}=\frac{1}{E\left(\sigma_{\gamma}\right)} ; \\
& a_{12}=a_{21}=-\frac{v\left(\sigma_{\alpha}\right)}{E\left(\sigma_{\alpha}\right)}=-\frac{v\left(\sigma_{\beta}\right)}{E\left(\sigma_{\beta}\right)} ; a_{13}=a_{31}=-\frac{v\left(\sigma_{\alpha}\right)}{E\left(\sigma_{\alpha}\right)}=-\frac{v\left(\sigma_{\gamma}\right)}{E\left(\sigma_{\gamma}\right)} ; \\
& a_{23}=a_{32}=-\frac{v\left(\sigma_{\beta}\right)}{E\left(\sigma_{\beta}\right)}=-\frac{v\left(\sigma_{\gamma}\right)}{E\left(\sigma_{\gamma}\right)} .
\end{aligned}
$$

In fact, we hold the assumption, proposed by Ambartsumyan [11,12], as follows

$$
a_{12}=a_{13}=a_{23}=-\frac{v^{+}}{E^{+}}=-\frac{v^{-}}{E^{-}} .
$$

For the sake of convenience of the formulation, (7) can be shorten as

$$
\begin{aligned}
& \sigma_{x}=2 A \varepsilon_{x}+B e-f_{11}\left(\sigma_{\alpha}, \sigma_{\beta}, \sigma_{\gamma}, m_{1}, m_{2}, m_{3}\right), \\
& \sigma_{y}=2 A \varepsilon_{y}+B e-f_{22}\left(\sigma_{\alpha}, \sigma_{\beta}, \sigma_{\gamma}, m_{1}, m_{2}, m_{3}\right), \\
& \sigma_{z}=2 A \varepsilon_{z}+B e-f_{33}\left(\sigma_{\alpha}, \sigma_{\beta}, \sigma_{\gamma}, m_{1}, m_{2}, m_{3}\right), \\
& \tau_{x y}=A \gamma_{x y}-f_{12}\left(\sigma_{\alpha}, \sigma_{\beta}, \sigma_{\gamma}, m_{1}, m_{2}, m_{3}\right), \\
& \tau_{y z}=A \gamma_{y z}-f_{23}\left(\sigma_{\alpha}, \sigma_{\beta}, \sigma_{\gamma}, m_{1}, m_{2}, m_{3}\right), \\
& \tau_{x z}=A \gamma_{x z}-f_{13}\left(\sigma_{\alpha}, \sigma_{\beta}, \sigma_{\gamma}, m_{1}, m_{2}, m_{3}\right),
\end{aligned}
$$

where the functions of stress state

$$
\begin{aligned}
& f_{11}=B_{3}\left(B+2 A m_{1}^{2}\right) \sigma_{\beta}, f_{22}=B_{3}\left(B+2 A m_{2}^{2}\right) \sigma_{\beta}, f_{33}=-B_{3}\left(B+2 A m_{3}^{2}\right) \sigma_{\beta}, \\
& f_{12}=2 A B_{3} m_{1} m_{2} \sigma_{\beta}, f_{23}=2 A B_{3} m_{2} m_{3} \sigma_{\beta}, f_{13}=2 A B_{3} m_{1} m_{3} \sigma_{\beta} .
\end{aligned}
$$

Substituting (16) into the equilibrium equation gives, with zero body forces, as

$$
\begin{aligned}
& A \nabla^{2} u+(A+B) \frac{\partial e}{\partial x}+2 \frac{\partial u}{\partial x} \frac{\partial A}{\partial x}+e \frac{\partial B}{\partial x}+\left(\frac{\partial u}{\partial y}+\frac{\partial v}{\partial x}\right) \frac{\partial A}{\partial y}+\left(\frac{\partial u}{\partial z}+\frac{\partial w}{\partial x}\right) \frac{\partial A}{\partial z}=\frac{\partial f_{11}}{\partial x}+\frac{\partial f_{12}}{\partial y}+\frac{\partial f_{13}}{\partial z} \\
& A \nabla^{2} v+(A+B) \frac{\partial e}{\partial y}+2 \frac{\partial v}{\partial y} \frac{\partial A}{\partial y}+e \frac{\partial B}{\partial y}+\left(\frac{\partial v}{\partial z}+\frac{\partial w}{\partial y}\right) \frac{\partial A}{\partial z}+\left(\frac{\partial v}{\partial x}+\frac{\partial u}{\partial y}\right) \frac{\partial A}{\partial x}=\frac{\partial f_{12}}{\partial x}+\frac{\partial f_{22}}{\partial y}+\frac{\partial f_{23}}{\partial z}
\end{aligned}
$$




$$
A \nabla^{2} w+(A+B) \frac{\partial e}{\partial z}+2 \frac{\partial w}{\partial z} \frac{\partial A}{\partial z}+e \frac{\partial B}{\partial z}+\left(\frac{\partial w}{\partial x}+\frac{\partial u}{\partial z}\right) \frac{\partial A}{\partial x}+\left(\frac{\partial w}{\partial y}+\frac{\partial v}{\partial z}\right) \frac{\partial A}{\partial y}=\frac{\partial f_{13}}{\partial x}+\frac{\partial f_{23}}{\partial y}+\frac{\partial f_{33}}{\partial z}
$$

where $\nabla^{2}=\partial^{2} / \partial x^{2}+\partial^{2} / \partial y^{2}+\partial^{2} / \partial z^{2}$. The traction boundary conditions, in terms of displacements are given as

$$
\begin{aligned}
& X=\operatorname{Ben}_{x}+A\left(\frac{\partial u}{\partial x} n_{x}+\frac{\partial u}{\partial y} n_{y}+\frac{\partial u}{\partial z} n_{z}\right)+A\left(\frac{\partial u}{\partial x} n_{x}+\frac{\partial v}{\partial x} n_{y}+\frac{\partial w}{\partial x} n_{z}\right)-\left(f_{11} n_{x}+f_{12} n_{y}+f_{13} n_{z}\right) \\
& Y=B e n_{y}+A\left(\frac{\partial v}{\partial x} n_{x}+\frac{\partial v}{\partial y} n_{y}+\frac{\partial v}{\partial z} n_{z}\right)+A\left(\frac{\partial u}{\partial y} n_{x}+\frac{\partial v}{\partial y} n_{y}+\frac{\partial w}{\partial y} n_{z}\right)-\left(f_{12} n_{x}+f_{22} n_{y}+f_{23} n_{z}\right) \\
& Z=B e n_{z}+A\left(\frac{\partial w}{\partial x} n_{x}+\frac{\partial w}{\partial y} n_{y}+\frac{\partial w}{\partial z} n_{z}\right)+A\left(\frac{\partial u}{\partial z} n_{x}+\frac{\partial v}{\partial z} n_{y}+\frac{\partial w}{\partial z} n_{z}\right)-\left(f_{13} n_{x}+f_{23} n_{y}+f_{33} n_{z}\right)
\end{aligned}
$$

where $X, Y$ and $Z$ are tractions on the boundary and $\mathbf{n}=\left(n_{x}, n_{y}, n_{z}\right)$ is the normal outward vector to the boundary. It is noticeable that the partial differential equations in (18), (19) and (20) with boundary conditions from (21) to (23) are non-linear as the coefficients $A, B$ and $f_{i j}$ are functions of the principal stress $\sigma_{\beta}$ and its directions $\left(m_{1}, m_{2}, m_{3}\right)$ as well.

\subsection{Plane stress problems}

For a two dimensional plane stress problem, all variables are independent of the $z$-axis and the principal directions $l_{3}=m_{3}=n_{1}=n_{2}=0, n_{3}=1$ and stresses $\sigma_{z}=\sigma_{\gamma}=\tau_{x z}=\tau_{y z}=0$. Therefore, Equations (16) and (17) can be simplified as

$$
\begin{aligned}
& \varepsilon_{x}=a_{11} \sigma_{x}+a_{12}\left(\sigma_{y}+\sigma_{z}\right)+B_{3} m_{1}^{2} \sigma_{\beta}, \\
& \varepsilon_{y}=a_{11} \sigma_{y}+a_{12}\left(\sigma_{z}+\sigma_{x}\right)+B_{3} m_{2}^{2} \sigma_{\beta}, \\
& \varepsilon_{z}=a_{12}\left(\sigma_{x}+\sigma_{y}\right)+B_{3} m_{3}^{2} \sigma_{\beta}, \\
& \gamma_{x y}=2 A_{1} \tau_{x y}+2 B_{3} m_{1} m_{2} \sigma_{\beta}, \\
& \gamma_{y z}=\gamma_{x z}=0
\end{aligned}
$$

or stresses can be written, in terms of strain, as

$$
\begin{aligned}
& \sigma_{x}=A\left[\left(\varepsilon_{x}-\lambda e\right)+B_{3}\left(\lambda-m_{1}^{2}\right) \sigma_{\beta}\right]=A \varepsilon_{x}-A \lambda e-f_{11}, \\
& \sigma_{y}=A\left[\left(\varepsilon_{y}-\lambda e\right)+B_{3}\left(\lambda-m_{2}^{2}\right) \sigma_{\beta}\right]=A \varepsilon_{x}-A \lambda e-f_{22}, \\
& \tau_{x y}=A\left[\gamma_{x y}-2 B_{3} m_{1} m_{2} \sigma_{\beta}\right]=\frac{A}{2} \gamma_{x y}-f_{12},
\end{aligned}
$$


where

$$
\begin{aligned}
& f_{11}=-A B_{3}\left(\lambda-m_{1}^{2}\right) \sigma_{\beta} ; f_{22}=-A B_{3}\left(\lambda-m_{1}^{2}\right) \sigma_{\beta} ; f_{12}=A B_{3} m_{1} m_{2} \sigma_{\beta}, \\
& \lambda=\frac{a_{12}}{a_{11}+a_{12}}, e=\varepsilon_{x}+\varepsilon_{y} .
\end{aligned}
$$

Then, the equilibrium equations yield

$$
\begin{aligned}
& \frac{A}{2} \nabla^{2} u+A\left(\frac{1}{2}-\lambda\right) \frac{\partial e}{\partial x}+\frac{\partial u}{\partial x} \frac{\partial A}{\partial x}-e \frac{\partial(\lambda A)}{\partial x}+\left(\frac{\partial v}{\partial x}+\frac{\partial u}{\partial y}\right) \frac{\partial A}{\partial y}=\frac{\partial f_{11}}{\partial x}+\frac{\partial f_{12}}{\partial y}, \\
& \frac{A}{2} \nabla^{2} u+A\left(\frac{1}{2}-\lambda\right) \frac{\partial e}{\partial y}+\frac{\partial v}{\partial y} \frac{\partial A}{\partial y}-e \frac{\partial(\lambda A)}{\partial y}+\left(\frac{\partial v}{\partial x}+\frac{\partial u}{\partial y}\right) \frac{\partial A}{\partial x}=\frac{\partial f_{12}}{\partial x}+\frac{\partial f_{22}}{\partial y},
\end{aligned}
$$

and two tractions on the boundary are

$$
\begin{aligned}
& X=-2 A \lambda\left(\frac{\partial u}{\partial x}+\frac{\partial v}{\partial y}\right) n_{x}+A\left(\frac{\partial u}{\partial x} n_{x}+\frac{\partial u}{\partial y} n_{y}\right)+A\left(\frac{\partial u}{\partial x} n_{x}+\frac{\partial v}{\partial x} n_{y}\right)-\left(f_{11} n_{x}+f_{12} n_{y}\right), \\
& Y=-2 A \lambda\left(\frac{\partial u}{\partial x}+\frac{\partial v}{\partial y}\right) n_{y}+A\left(\frac{\partial v}{\partial x} n_{x}+\frac{\partial v}{\partial y} n_{y}\right)+A\left(\frac{\partial u}{\partial y} n_{x}+\frac{\partial v}{\partial y} n_{y}\right)-\left(f_{12} n_{x}+f_{22} n_{y}\right) .
\end{aligned}
$$

For the sake of convenience of analysis, we assume that $\sigma_{\alpha}>\sigma_{\beta}$ and hold

$$
m_{1}^{2}=\frac{1}{1+k^{2}}, m_{2}^{2}=\frac{k^{2}}{1+k^{2}}, m_{1} m_{2}=\frac{k}{1+k^{2}},
$$

where

$$
k=-t \pm \sqrt{1+t^{2}}, t=\frac{\sigma_{x}-\sigma_{y}}{2 \tau_{x y}} .
$$

\section{Meshless finite block method and iterative algorithm}

\subsection{Lagrange polynomial interpolation}

By using the Lagrange polynomial interpolation, the function $u(Q)$ at $Q(\xi, \eta)$ can be approximated as

$$
u(Q)=\sum_{i=1}^{N_{\xi}} \sum_{j=1}^{N_{\eta}} F\left(\xi, \xi_{i}\right) G\left(\eta, \eta_{j}\right) u^{(k)}=\sum_{k=1}^{M} \phi_{k}(Q) u^{(k)}
$$

where 


$$
F\left(\xi, \xi_{i}\right)=\prod_{\substack{m=1 \\ m \neq i}}^{N_{\xi}} \frac{\left(\xi-\xi_{m}\right)}{\left(\xi_{i}-\xi_{m}\right)}, G\left(\eta, \eta_{j}\right)=\prod_{\substack{n=1 \\ n \neq j}}^{N_{\eta}} \frac{\left(\eta-\eta_{n}\right)}{\left(\eta_{j}-\eta_{n}\right)}
$$

For two dimensional problems, a set of nodes in the normalized domain are considered, as shown in Figure 5. At $P\left(\xi_{k}, \eta_{k}\right), k=(j-1) \times N_{\xi}+i, i=1,2, \ldots, N_{\xi}$ and $j=1,2, \ldots, N_{\eta}$, where $N_{\alpha}(\alpha=\xi, \eta)$ indicate the node numbers along the two axes and the total number of nodes $M=N_{\xi} \times N_{\eta}$. It is easier to use shape functions in the following analysis as

$$
\phi_{k}(Q)=F\left(\xi, \xi_{i}\right) G\left(\eta, \eta_{j}\right)=\prod_{\substack{m=1 \\ m \neq i}}^{N_{\xi}} \frac{\left(\xi-\xi_{m}\right)}{\left(\xi_{i}-\xi_{m}\right)} \prod_{\substack{n=1 \\ n \neq j}}^{N_{\eta}} \frac{\left(\eta-\eta_{n}\right)}{\left(\eta_{j}-\eta_{n}\right)}
$$

Therefore, the first order partial differential of the shape function $\phi_{k}(Q)$ can be determined easily as

$$
\frac{\partial \phi_{k}(Q)}{\partial \xi}=\frac{\partial F\left(\xi, \xi_{i}\right)}{\partial \xi} G\left(\eta, \eta_{j}\right), \frac{\partial \phi_{k}(Q)}{\partial \eta}=F\left(\xi, \xi_{i}\right) \frac{\partial G\left(\eta, \eta_{j}\right)}{\partial \eta}
$$

where

$$
\frac{\partial F}{\partial \xi}=\sum_{l=1}^{N_{\xi}} \prod_{i=1, i \neq i, i \neq l}^{N_{\xi}}\left(\xi-\xi_{i}\right) / \prod_{m=1, m \neq i}^{N_{\xi}}\left(\xi_{i}-\xi_{m}\right)
$$

and

$$
\frac{\partial G}{\partial \eta}=\sum_{l=1}^{N_{\eta}} \prod_{j=1, j \neq i, j \neq l}^{N_{\eta}}\left(\eta-\eta_{j}\right) / \prod_{n=1, n \neq j}^{N_{\eta}}\left(\eta_{j}-\eta_{n}\right)
$$

\subsection{Partial differential matrix}

The nodal value of the first order partial differentials can be written in a vector form from (32), in terms of the nodal value of function $u$, see [34,35], as

$$
\mathbf{u}_{, \alpha}=\mathbf{U}_{\alpha}=\mathbf{D}_{\alpha} \mathbf{u}, \mathbf{D}_{\alpha}=\left\{\phi_{k l, \alpha}\right\}_{M \times M}, \quad(k, l=1,2, \ldots, M ; \alpha=\xi, \eta),
$$

where

$$
\mathbf{u}_{, \alpha}=\left\{\frac{\partial u\left(P_{1}\right)}{\partial \alpha}, \frac{\partial u\left(P_{2}\right)}{\partial \alpha}, \ldots, \frac{\partial u\left(P_{M}\right)}{\partial \alpha}\right\}^{\mathrm{T}}, \mathbf{u}=\left\{u\left(P_{1}\right), u\left(P_{2}\right), \ldots, u\left(P_{M}\right)\right\}^{\mathrm{T}} .
$$

The $L$-th order partial differentials in two dimensions with respect to both coordinates $\xi$ and $\eta$ can be obtained approximately by 


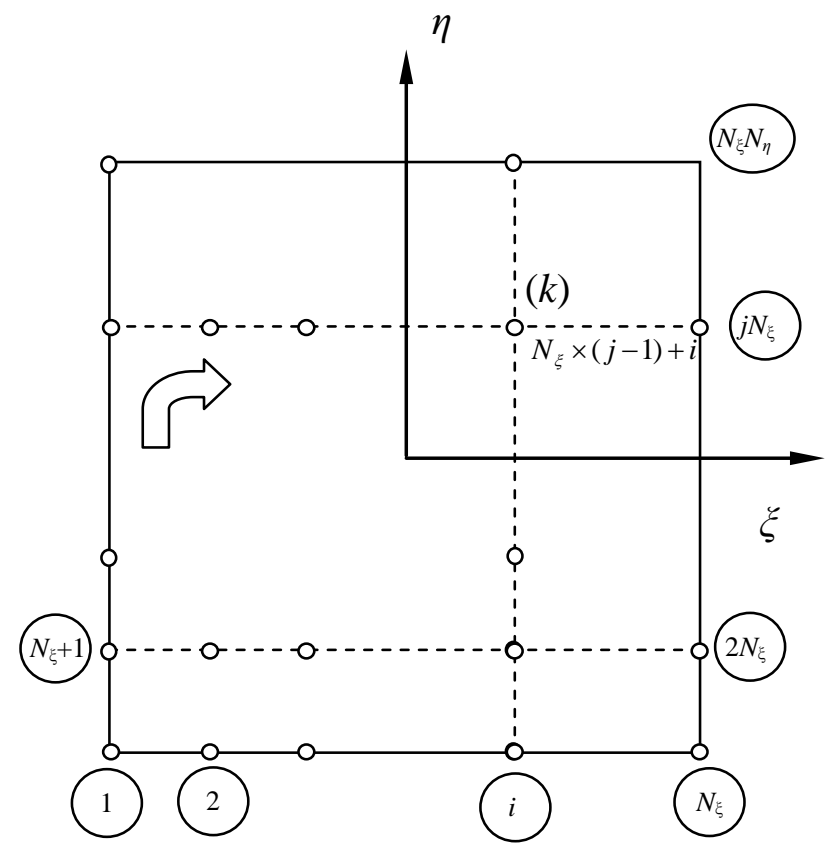

Figure 5. Uniformly distributed nodes in mapped domain and numbering system for FBM.

$$
u_{, m n}(\xi, \eta)=\frac{\partial^{m+n} u}{\partial \xi^{m} \partial \eta^{n}}, \quad m+n=L
$$

Therefore, the vectors of the higher order partial differentials can be constructed [34,35], in terms of the first order partial differential matrices $\mathbf{D}_{\xi}$ and $\mathbf{D}_{\eta}$, as

$$
\mathbf{u}_{, m n} \approx \mathbf{D}_{\xi}^{m} \mathbf{D}_{\eta}^{n} \mathbf{u}
$$

\subsection{Mapping differential matrix}

A quadratic block with 8 seeds shown in Figure 6(a) is mapped into a normalized domain (square) as shown in Figure 6(b) using the shape functions

$$
\begin{aligned}
& N_{i}=\frac{1}{4}\left(1+\bar{\xi}_{i} \xi\right)\left(1+\overline{\eta_{i}} \eta\right)\left(\bar{\xi}_{i} \xi+\bar{\eta}_{i} \eta-1\right) \text { for } i=1,2,3,4 \\
& N_{i}=\frac{1}{2}\left(1-\xi^{2}\right)\left(1+\bar{\eta}_{i} \eta\right) \text { for } i=5,7 \\
& N_{i}=\frac{1}{2}\left(1-\eta^{2}\right)\left(1+\bar{\xi}_{i} \xi\right) \text { for } i=6,8
\end{aligned}
$$




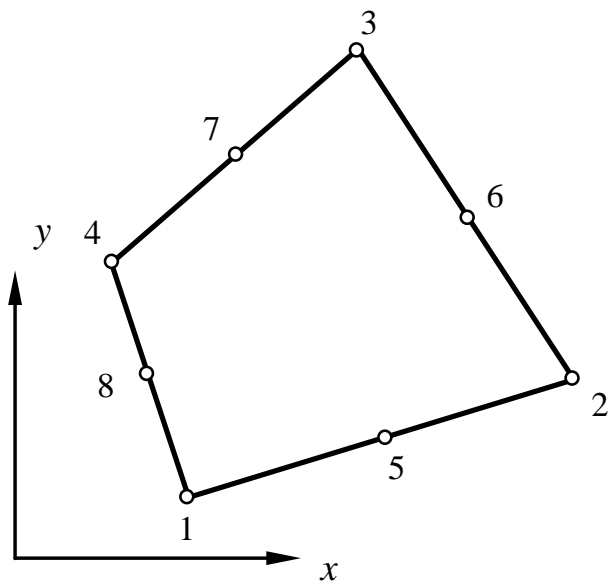

(a)

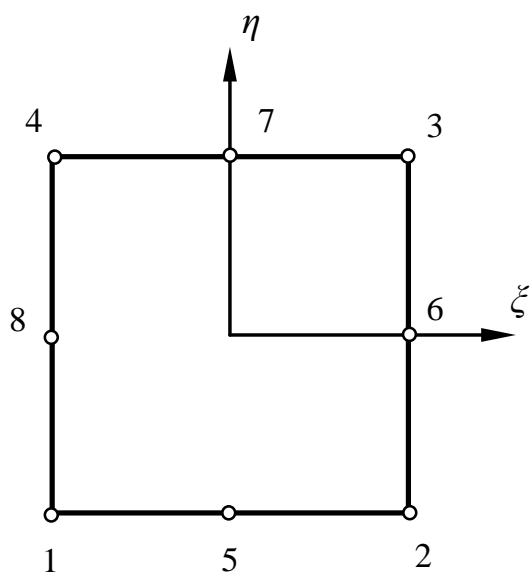

(b)

Figure 6. Five nodes mapping: (a) practical domain; (b)normalized domain.

where $\left(\bar{\xi}_{i}, \bar{\eta}_{i}\right)(i=1,2, \ldots, 8)$ are the seed coordinates shown in Figure 4(b). Therefore, the transformation of the coordinate (mapping) for the real domain can be written as

$$
x=\sum_{k=1}^{8} N_{k}(\xi, \eta) x_{k}, y=\sum_{k=1}^{8} N_{k}(\xi, \eta) y_{k}
$$

The partial differentials of the function $u(x, y)$ is given by

$$
\frac{\partial u}{\partial x}=\frac{1}{J}\left(\beta_{11} \frac{\partial u}{\partial \xi}+\beta_{12} \frac{\partial u}{\partial \eta}\right), \frac{\partial u}{\partial y}=\frac{1}{J}\left(\beta_{21} \frac{\partial u}{\partial \xi}+\beta_{22} \frac{\partial u}{\partial \eta}\right),
$$

where

$$
J=\left|\begin{array}{ll}
\frac{\partial x}{\partial \xi} & \frac{\partial x}{\partial \eta} \\
\frac{\partial y}{\partial \xi} & \frac{\partial y}{\partial \eta}
\end{array}\right|, \quad \beta_{11}=\frac{\partial y}{\partial \eta}, \beta_{12}=-\frac{\partial y}{\partial \xi}, \beta_{21}=-\frac{\partial x}{\partial \eta}, \beta_{22}=\frac{\partial x}{\partial \xi} .
$$

Therefore, the first order partial differential matrices in the physical domain can be obtained

$$
\begin{aligned}
& \mathbf{U}_{x}=\Delta_{11} \mathbf{U}_{\xi}+\Delta_{12} \mathbf{U}_{\eta}=\left(\boldsymbol{\Delta}_{11} \mathbf{D}_{\xi}+\Delta_{12} \mathbf{D}_{\eta}\right) \mathbf{u}=\mathbf{D}_{x} \mathbf{u}, \\
& \mathbf{U}_{y}=\boldsymbol{\Delta}_{21} \mathbf{U}_{\xi}+\boldsymbol{\Delta}_{22} \mathbf{U}_{\eta}=\left(\boldsymbol{\Delta}_{21} \mathbf{D}_{\xi}+\boldsymbol{\Delta}_{22} \mathbf{D}_{\eta}\right) \mathbf{u}=\mathbf{D}_{y} \mathbf{u},
\end{aligned}
$$

in which 


$$
\boldsymbol{\Delta}_{i j}=\left(\begin{array}{cccc}
\beta_{i j}^{(1)} / J^{(1)} & 0 & \ldots & 0 \\
0 & \beta_{i j}^{(2)} / J^{(2)} & \ldots & 0 \\
\ldots & \ldots & \ldots & \ldots \\
0 & 0 & \ldots & \beta_{i j}^{(M)} / J^{(M)}
\end{array}\right) \text {, }
$$

$\beta_{i j}^{(k)} / J^{(k)}$ is calculated from (45) at collocation point $P_{k}=\left(\xi_{k}, \eta_{k}\right)$. It is apparent that the nodal values of the first order partial differentials can be determined in terms of the first order partial differential matrix in the normalized domain, where $|\xi| \leq 1 ;|\eta| \leq 1$.

\subsection{Iterative algorithm with meshless method}

Meshless finite block method has two approaches including the formations of the governing equation in a strong form and a weak form. Both solutions have different advantages as discussed by Wen and Cao [34], and Li et al [35]. The formulations in a strong form are simpler and more direct. The equilibrium equations in terms of displacements from (28) give

$$
\begin{aligned}
& (1-\lambda) A \frac{\partial^{2} u}{\partial x^{2}}+A \frac{\partial^{2} u}{\partial y^{2}}+\left(\frac{1}{2}-\lambda\right) A \frac{\partial^{2} v}{\partial x \partial y}+\frac{\partial u}{\partial x} \frac{\partial A}{\partial x}-e \frac{\partial(\lambda A)}{\partial x}+\left(\frac{\partial v}{\partial x}+\frac{\partial u}{\partial y}\right) \frac{\partial A}{\partial y}=b_{x} \\
& \left(\frac{1}{2}-\lambda\right) A \frac{\partial^{2} u}{\partial x \partial y}+(1-\lambda) A \frac{\partial^{2} v}{\partial y^{2}}+A \frac{\partial^{2} v}{\partial x^{2}}+\frac{\partial v}{\partial y} \frac{\partial A}{\partial y}-e \frac{\partial(\lambda A)}{\partial y}+\left(\frac{\partial v}{\partial x}+\frac{\partial u}{\partial y}\right) \frac{\partial A}{\partial x}=b_{y}
\end{aligned}
$$

where the body forces on the right hand side

$$
b_{x}=\frac{\partial f_{11}}{\partial x}+\frac{\partial f_{12}}{\partial y}, \quad b_{y}=\frac{\partial f_{12}}{\partial x}+\frac{\partial f_{22}}{\partial y} .
$$

They are non-linear partial differential equations due to the variables $\lambda, b_{x}$ and $b_{y}$ dependent on the stress state $\left(\sigma_{x}, \sigma_{y}, \tau_{x y}\right)$. Applying partial differential matrices over (48) produce

$$
\begin{aligned}
& \mathbf{A}\left[(\mathbf{I}-\lambda) \mathbf{D}_{x}^{2}+\mathbf{D}_{y^{\prime}}^{2}\right] \mathbf{u}+\mathbf{A}\left(\frac{1}{2} \mathbf{I}-\lambda\right) \mathbf{D}_{x} \mathbf{D}_{y} \mathbf{v}+\frac{\partial \mathbf{A}}{\partial x} \mathbf{D}_{x} \mathbf{u}-\frac{\partial(\lambda \mathbf{A})}{\partial x}\left(\mathbf{D}_{x} \mathbf{u}+\mathbf{D}_{y} \mathbf{v}\right)+\frac{\partial \mathbf{A}}{\partial y}\left(\mathbf{D}_{y} \mathbf{u}+\mathbf{D}_{x} \mathbf{v}\right) \\
& =\mathbf{D}_{x} \mathbf{f}_{11}+\mathbf{D}_{y} \mathbf{f}_{12} \\
& \mathbf{A}\left(\frac{1}{2} \mathbf{I}-\boldsymbol{\lambda}\right) \mathbf{D}_{y} \mathbf{D}_{x} \mathbf{u}+\mathbf{A}\left[(\mathbf{I}-\boldsymbol{\lambda}) \mathbf{D}_{y}^{2}+\mathbf{D}_{x^{\prime}}^{2}\right] \mathbf{v}+\frac{\partial \mathbf{A}}{\partial y} \mathbf{D}_{y} \mathbf{v}-\frac{\partial(\lambda \mathbf{A})}{\partial y}\left(\mathbf{D}_{y} \mathbf{v}+\mathbf{D}_{x} \mathbf{u}\right)+\frac{\partial \mathbf{A}}{\partial x}\left(\mathbf{D}_{x} \mathbf{v}+\mathbf{D}_{y} \mathbf{u}\right) \\
& =\mathbf{D}_{x} \mathbf{f}_{12}+\mathbf{D}_{y} \mathbf{f}_{22}
\end{aligned}
$$


where $\mathbf{u}^{\mathrm{T}}=\left\{u_{1}, u_{2}, \ldots, u_{M}\right\}, \mathbf{v}^{\mathrm{T}}=\left\{v_{1}, v_{2}, \ldots, v_{M}\right\}, \mathbf{f}_{i j}^{\mathrm{T}}=\left\{f_{i j 1}, f_{i j}, \ldots, f_{i j M}\right\}$ are vectors of nodal value, $\mathbf{I}$ is the unit diagonal matrix and diagonal matrices defined as following

$$
\begin{aligned}
& \lambda=\left(\begin{array}{cccc}
\lambda_{1} & 0 & \ldots & 0 \\
0 & \lambda_{2} & \ldots & 0 \\
\ldots & \ldots & \ldots & \ldots \\
0 & 0 & \ldots & \lambda_{M}
\end{array}\right), \mathbf{A}=\left(\begin{array}{cccc}
A_{1} & 0 & \ldots & 0 \\
0 & A_{2} & \ldots & 0 \\
\ldots & \ldots & \ldots & \ldots \\
0 & 0 & \ldots & A_{M}
\end{array}\right), \\
& \frac{\partial(\lambda \mathbf{A})}{\partial x}=\left(\begin{array}{cccc}
\left.\frac{\partial(\lambda A)}{\partial x}\right|_{1} & 0 & \ldots & 0 \\
0 & \left.\frac{\partial(\lambda A)}{\partial x}\right|_{2} & \ldots & 0 \\
\ldots & \ldots & \ldots & \ldots \\
0 & 0 & \ldots & \left.\frac{\partial(\ddot{\lambda A})}{\partial x}\right|_{M}
\end{array}\right)
\end{aligned}
$$

The traction boundary conditions at the boundary point $P\left(x_{k}, y_{k}\right)$ can be written from (29) as

$$
\begin{aligned}
& X_{k}=A_{k} \sum_{l=1}^{M}\left(2(1-\lambda) n_{x} D_{x}^{k l}+n_{y} D_{y}^{k l}\right) u_{l}+A_{k} \sum_{l=1}^{M}\left(n_{y} D_{x}^{k l}-2 \lambda n_{x} D_{y}^{k l}\right) \nu_{l}-f_{11} n_{x}-f_{12} n_{y} \\
& Y_{k}=A_{k} \sum_{l=1}^{M}\left(2(1-\lambda) n_{y} D_{y}^{k l}+n_{x} D_{x}^{k l}\right) \nu_{l}+A_{k} \sum_{l=1}^{M}\left(n_{x} D_{y}^{k l}-2 \lambda n_{y} D_{x}^{k l}\right) u_{l}-f_{12} n_{x}-f_{22} n_{y}
\end{aligned}
$$

The iterative algorithm used by the meshless finite block method can be fulfilled with the following procedures

Step 1: Set $m=0$ and all elastic parameters are specified as $E^{+}=E^{-}=E, v^{+}=v^{-}=v$ and $\lambda=-\frac{v}{1-v}$. All non-linear coefficients $f_{i j}^{0}$ are set to be zero. The displacements $\left(u^{0}, v^{0}\right)$ and stresses $\left(\sigma_{x}^{0}, \sigma_{y}^{0}, \tau_{x y}^{0}\right)$ are obtained by solving (50) and (51) with boundary conditions (SM);

Step 2: Determine the principal stresses $\left(\sigma_{\beta}^{0}\right)$ and their directions $\left(m_{1}^{0}, m_{2}^{0}\right)$ coefficients, including $\lambda^{0}, A^{0}, f_{i j}^{0}$ which are functions of the principal stresses $\left(\sigma_{\alpha}^{0}, \sigma_{\beta}^{0}\right)$ and the stress state $\left(\sigma_{x}^{0}, \sigma_{y}^{0}, \tau_{x y}^{0}\right)$ at each collocation point;

Step 3: Set $m=m+1$;

Step 4: Determine displacements $\left(u^{m}, v^{m}\right)$ and stresses $\left(\sigma_{x}^{m}, \sigma_{y}^{m}, \tau_{x y}^{m}\right)$ by solving the following linear algebraic systems equation with boundary conditions, 


$$
\begin{aligned}
& \mathbf{A}^{m-1}\left[\left(\mathbf{I}-\lambda^{m-1}\right) \mathbf{D}_{x}^{2}+\mathbf{D}_{y^{\prime}}^{2}\right] \mathbf{u}^{m}+\mathbf{A}^{m-1}\left(\frac{1}{2} \mathbf{I}-\lambda^{m-1}\right) \mathbf{D}_{x} \mathbf{D}_{y} \mathbf{v}^{m}+\frac{\partial \mathbf{A}^{m-1}}{\partial x} \mathbf{D}_{x} \mathbf{u}^{m} \\
& -\frac{\partial(\lambda \mathbf{A})^{m-1}}{\partial x}\left(\mathbf{D}_{x} \mathbf{u}^{m}+\mathbf{D}_{y} \mathbf{v}^{m}\right)+\frac{\partial \mathbf{A}^{m-1}}{\partial y}\left(\mathbf{D}_{y} \mathbf{u}^{m}+\mathbf{D}_{x} \mathbf{v}^{m}\right)=\mathbf{b}_{x}^{m-1} \\
& \mathbf{A}^{m-1}\left(\frac{1}{2} \mathbf{I}-\lambda^{m-1}\right) \mathbf{D}_{y} \mathbf{D}_{x} \mathbf{u}^{m}+\mathbf{A}^{m-1}\left[\left(\mathbf{I}-\lambda^{m-1}\right) \mathbf{D}_{y}^{2}+\mathbf{D}_{x}^{2}\right] \mathbf{v}^{m}+\frac{\partial \mathbf{A}^{m-1}}{\partial y} \mathbf{D}_{y} \mathbf{v}^{m} \\
& -\frac{\partial(\lambda \mathbf{A})^{m-1}}{\partial y}\left(\mathbf{D}_{y} \mathbf{v}^{m}+\mathbf{D}_{x} \mathbf{u}^{m}\right)+\frac{\partial \mathbf{A}^{m-1}}{\partial x}\left(\mathbf{D}_{x} \mathbf{v}^{m}+\mathbf{D}_{y} \mathbf{u}^{m}\right)=\mathbf{b}_{y}^{m-1}
\end{aligned}
$$

Step 5: Determine the principal stresses $\left(\sigma_{\beta}^{m}\right)$ and their directions $\left(m_{1}^{m}, m_{2}^{m}\right)$, all coefficients including $\lambda^{m}, A^{m}, f_{i j}^{m}$ by stress state $\left(\sigma_{x}, \sigma_{y}, \tau_{x y}\right)$ at each collocation point. To speed up the iterative process, the vectors of the body force are modified by

$$
\begin{aligned}
& \mathbf{b}_{x}^{m}=\alpha\left(\mathbf{D}_{x} \mathbf{f}_{11}^{m}+\mathbf{D}_{y} \mathbf{f}_{12}^{m}\right)+(1-\alpha)\left(\mathbf{D}_{x} \mathbf{f}_{11}^{m-1}+\mathbf{D}_{y} \mathbf{f}_{12}^{m-1}\right) \\
& \mathbf{b}_{y}^{m}=\alpha\left(\mathbf{D}_{x} \mathbf{f}_{12}^{m}+\mathbf{D}_{y} \mathbf{f}_{22}^{m}\right)+(1-\alpha)\left(\mathbf{D}_{x} \mathbf{f}_{12}^{m-1}+\mathbf{D}_{y} \mathbf{f}_{22}^{m-1}\right)
\end{aligned},
$$

where $\alpha$ is the modification factor in the iterative process. In the computational procedure, the average value of the body force term is considered, and $\alpha=0.5$. Evidently, the selection of the modification factor does not have much influence on the final numerical results;

Step 6: Check the average error of displacement for all collocations $\eta$

$$
\eta=\frac{1}{M} \sum_{l=1}^{M}\left|u_{l}^{m}-u_{l}^{m-1}\right|
$$

if $\eta<10^{-6}$ go to Step 8

Step 7: set $m=m+1$ and go to Step 4;

Step 8: Print results and the computation is terminated.

\section{Numerical analysis}

\subsection{Cantilever beam in bimodular material}

First, we consider a beam of length $L$ and height $h$ with bimodular material loaded by a linear distributed normal stress shown in Figure 7. It is assumed that $\tau_{x y}=0$ and the normal stress $\sigma_{x}$ can be derived in the following, by the assumption [11], as 


$$
\varepsilon_{x}=\frac{y^{\prime}}{\rho}
$$

where $\rho$ is the radius of curvature and $y^{\prime}$ is measured from the neutral axis. Therefore, we have

$$
\sigma_{x}=\left\{\begin{array}{ll}
E^{+} \frac{y^{\prime}}{\rho} & \text { if } 0<y^{\prime} \leq h_{1} \\
E^{-} \frac{y^{\prime}}{\rho} & \text { if }-h_{2} \leq y^{\prime}<0
\end{array} .\right.
$$

From the equilibrium conditions, one has

$$
h_{1}=\frac{h \sqrt{E^{-}}}{\sqrt{E^{+}}+\sqrt{E^{-}}}, h_{1}=\frac{h \sqrt{E^{+}}}{\sqrt{E^{+}}+\sqrt{E^{-}}}
$$

and

$$
\frac{1}{\rho}=\frac{M}{D} \text {, where } D=\frac{b}{3}\left(E^{-} h_{2}^{3}+E^{+} h_{1}^{3}\right)
$$

In the following numerical simulations, the ratio of Young's modulus $E^{-} / E^{+}=2$ and Poisson ratio $v^{+}=0.1$. All computations are performed with double precision on a Lenovo-PC. The ratio $L / h=2$ and one block is used, and the node numbers $N_{\xi}=2 N_{\eta}$. The location of nodes along the two axes in the normalized domain is selected as

$$
\xi_{i}=-\cos \frac{\pi(i-1)}{N_{\xi}-1}, i=1,2, \ldots, N_{\xi} ; \quad \eta_{j}=-\cos \frac{\pi(j-1)}{N_{\eta}-1}, j=1,2, \ldots, N_{\eta}
$$

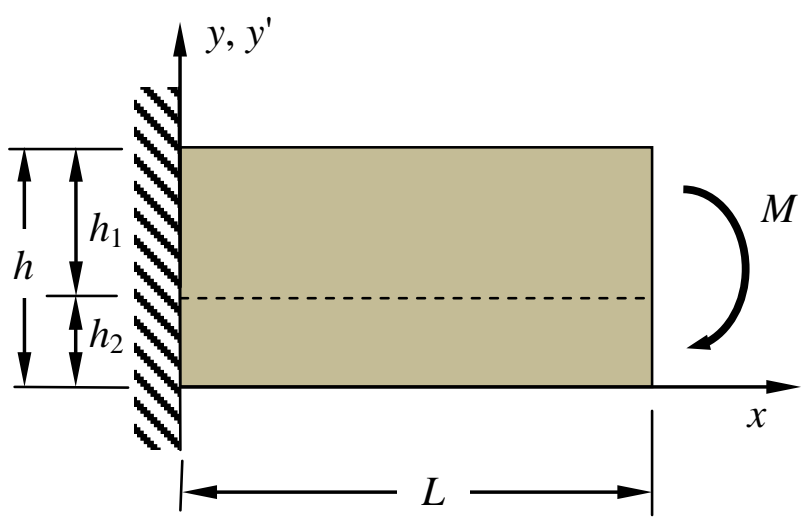

Figure 7. Cantilever beam in bimodular material. 


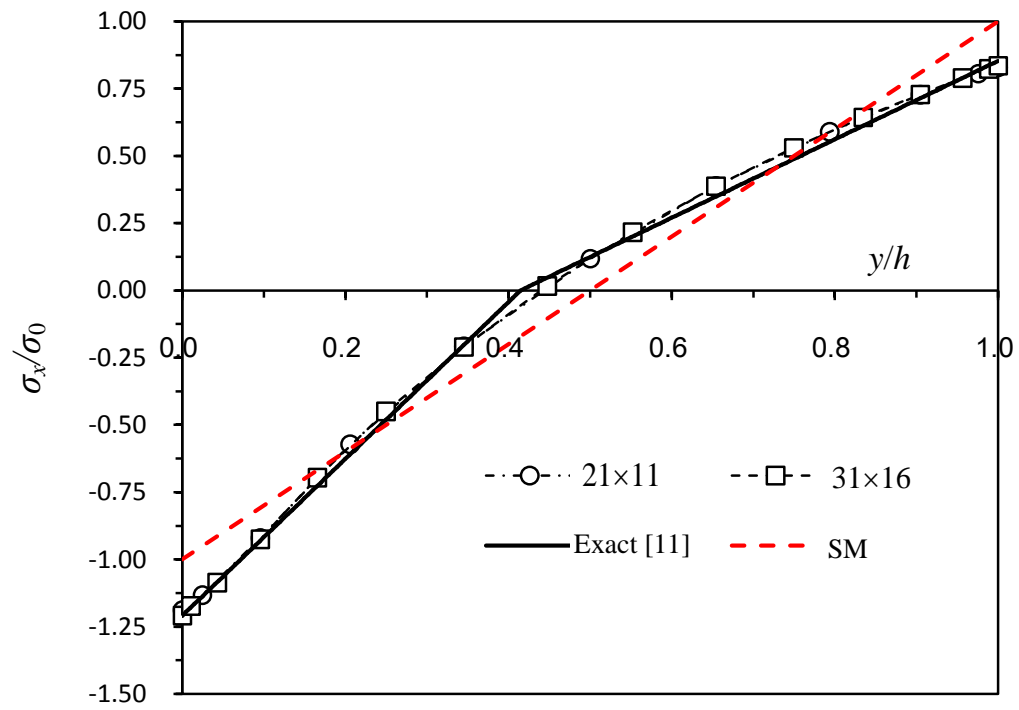

Figure 8. Normal stress distribution $\sigma_{x} / \sigma_{0}$ on the cross section $x=L / 2$ with characteristic parameter $\beta=0.1$.

The normalized stress distribution $\sigma_{x} / \sigma_{0}$ at $x=0.5 \mathrm{~L}$ for linear smooth mode of Young's modulus is shown in Figure 8 where the characteristic factor of material $\beta=0.1$ and the node number is selected as $N_{\eta}=11$ or $N_{\eta}=16$. Analytical solution in (60) is presented in the figure to show the degrees of accuracy and convergence of this method. The bending normal stress for the Same Modulus (SM) material, i.e. $E^{-} / E^{+}=1$, is presented to show the influence of the bimodular material. It can be seen that the neutral axis shifts to the bottom and the value of normal compressive stress is larger than tensile stress due to the bimodular property.

In order to determine the degree of accuracy for any numerical method, a rigorous study of the numerical error and a convergence analysis must be conducted. Due to the difficulty in evaluating a high nonlinear problem, it was very challenging to estimate the error for the FBM in this context. Nonetheless, the accuracy and efficiency of the numerical results is provided in this example. Table 1 shows the average error for normal stress between the numerical results and the analytical solutions, i.e. $\eta=\sum_{l=1}^{N_{\eta}}\left|\sigma_{x}^{(l)}-\sigma_{x}^{*(l)}\right| / N_{\eta}$, where $\sigma_{x}^{*(l)}$ is the analytical solution defined in (60) for the different models and characteristic factor $\beta$. It is shown that the influence of the characteristic factor $\beta$ is significant for both material models. In general, the aver- 
age error decreases with an increase in the node density. It is expected that the error is larger for a small characteristic factor. In addition, the common logarithm of the average error versus the iteration step is shown in Figure 9 when $N_{\eta}=16$. For the iteration process, it can be seen that the results converged for a large range of characteristic factor selection, i.e. $\beta \geq 0.01$. It is true that a converge result can be achieved quickly with an average error of 1\% at iteration step 5 for each characteristic factor $\beta$. However, the numerical solutions are affected significantly when $\beta<0.01$ and the oscillation of the results is observed for large iterative number $(m>8)$.

Table 1. Average errors with different modes and character parameters.

\begin{tabular}{|c|c|c|c|c|c|c|}
\hline \multirow{2}{*}{$N_{\xi} \times N_{\eta}$} & \multicolumn{2}{|c|}{$\beta=1.0$} & \multicolumn{2}{c|}{$\beta=0.1$} & \multicolumn{2}{c|}{$\beta=0.01$} \\
\cline { 2 - 7 } & Linear & Hyperbolic & Linear & Hyperbolic & Linear & Hyperbolic \\
\hline $21 \times 11$ & 0.0018 & 0.0030 & 0.0023 & 0.0190 & 0.0259 & 0.0216 \\
\hline $31 \times 16$ & 0.0014 & 0.0022 & 0.0021 & 0.0125 & 0.0064 & 0.0093 \\
\hline
\end{tabular}

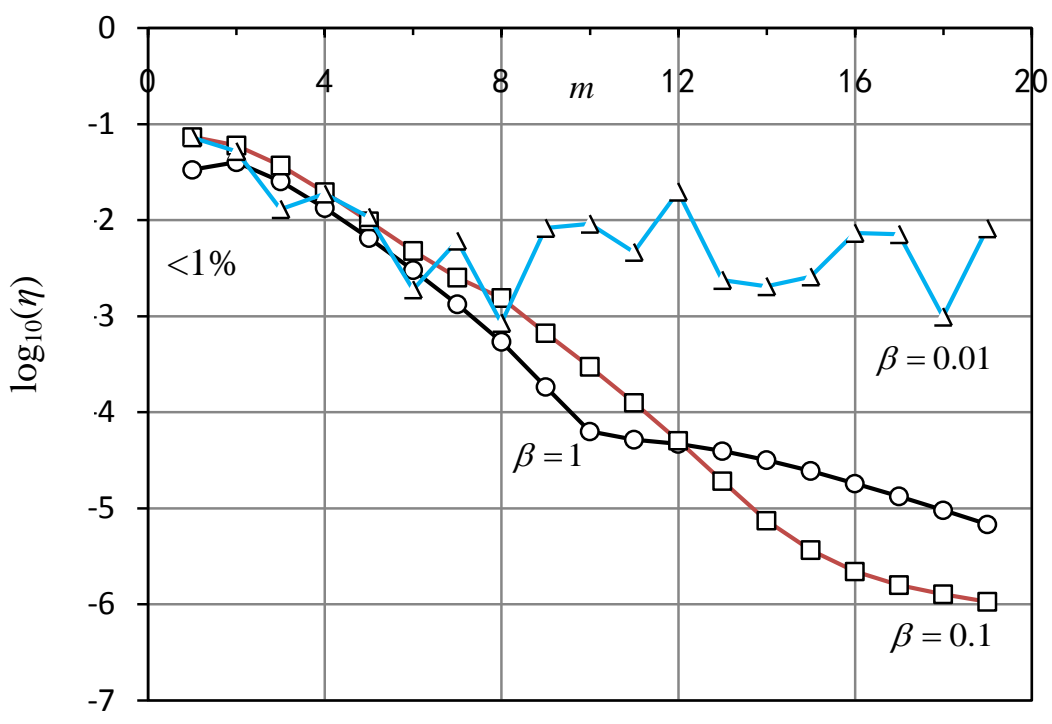

Figure 9. The common logarithm of average error in (58) against the iteration number $m$ for different characteristic factor $\beta$. 


\subsection{Square plate with circular hole under pressure load}

Secondly, a square plate containing a circular hole as shown in Figure 10(a) subjected to a pressure load $\sigma_{0}$ is considered. Due to the symmetry of the problem, only a quarter of the plate is modelled with half the width $a=2 R$ with two blocks. It is clear that a minimum number of two blocks is required for this configuration of the domain. For a complicated problem including contact between two bodies [36] or a cracked body [37], more blocks should be used. In order to show the distribution of the collocation points in the physical domain as in Figure 10(b), the node numbers $N_{\xi} \times N_{\eta}=11 \times 11$ are selected with regular distribution in the normalized domain. On the interface, both continuous conditions of displacement and traction $\left(\eta^{I}=+1\right.$ and $\left.\eta^{I I}=-1\right)$ have to be satisfied, i.e. $u^{I}=u^{I I}, v^{I}=v^{I I}, t_{x}^{I}+t_{x}^{I I}=0$ and $t_{y}^{I}+t_{y}^{I I}=0$.

The number of nodes $\left(N_{\xi}^{I} \times N_{\eta}^{I}\right)$ and $\left(N_{\xi}^{I I} \times N_{\eta}^{I I}\right)$ are equal and selected to be 16 on each axis and the hyperbolic tangent model is adopted.

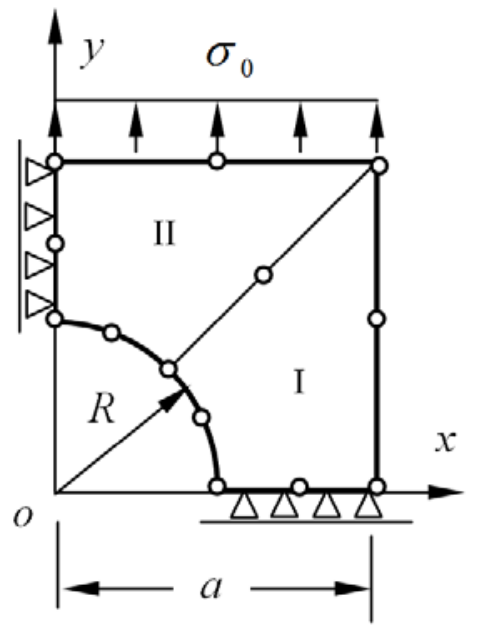

(a)

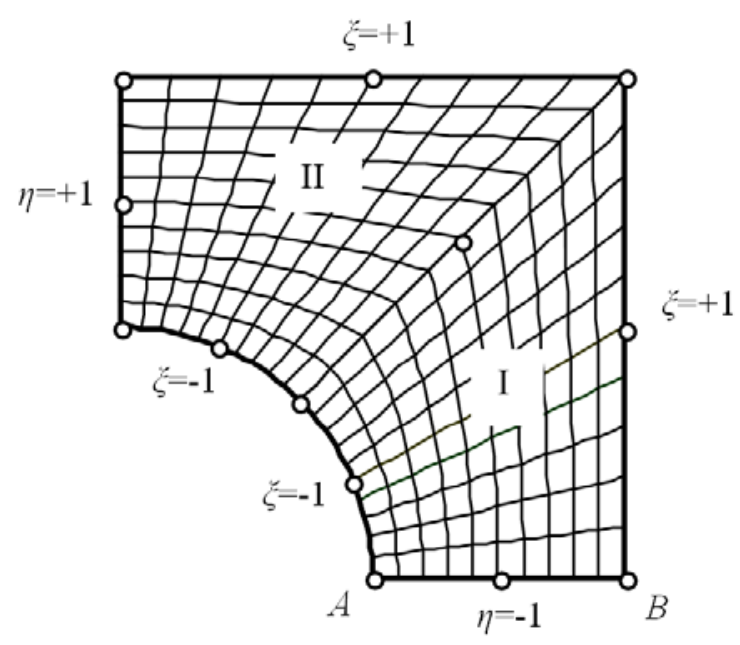

(b)

Figure 10. Blocks for a quarter square plate and their mapping seeds (a) geometry and boundary conditions; (b) distribution of node in two blocks.

To show the efficiency and accuracy of the meshless method, the numerical solutions given by FEM [38] are also shown on the same figure for comparison. By comparing the meshless FBM method to the FEM method, two main advantages of the proposed method can be ob- 
served. First, it can be said that the FBM modeling of the problem is very simple when compared to the FEM method. The elements used in meshing the rectangular plate and the solid cylinder are shown in Figure 11. In this example the total number of nodes used by the FBM method is 242 and by the FEM method is 13128 . To achieve a highly accurate numerical solution with the FEM method, a high density mesh should be considered. Secondly, the convergence of the FBM method can be achieved easily. For the FEM method, several special algorithms must be applied in the iteration procedure in order to obtain a convergent result as described in [38]. Figures 12(a)(b) show the normalized stress distributions $\sigma_{y} / \sigma_{0}$ and $\sigma_{x} / \sigma_{0}$ on edges $\mathrm{AB}$ and $\mathrm{BC}$ respectively when characteristic factor $\beta$ is taken to be 0.5 . The solutions for the same modulus (SM) material are presented to show the gaps caused by bimodular property of the material. Also, very small influence can be found caused by characteristic factor $\beta$ as shown in Figures 13(a)(b) in the region $5 \leq \beta \leq 0.5$. Similar to the previous example, the instability with oscillation of results in iteration occurs when $\beta \leq 0.1$ and $m>6$. An excellent agreement with FEM was achieved from the numerical results in these figures.

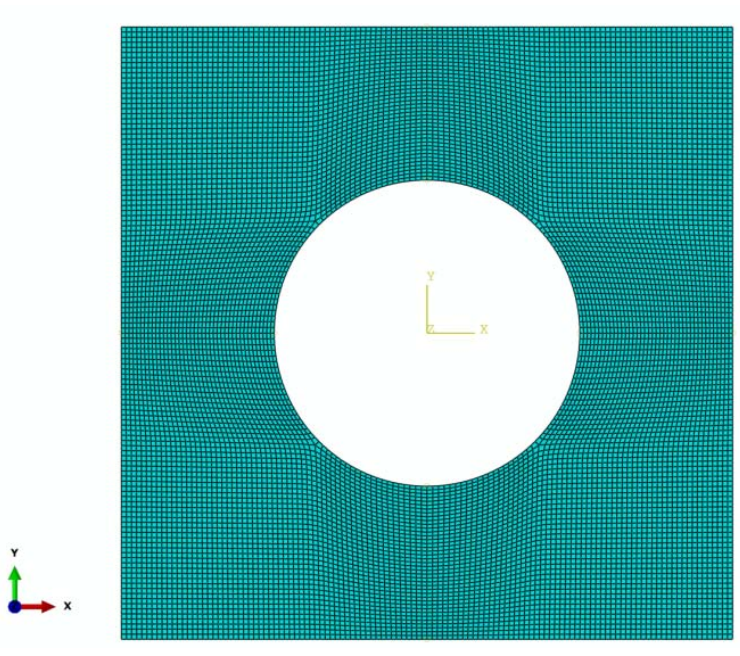

Elements:12804, nodes: 13128

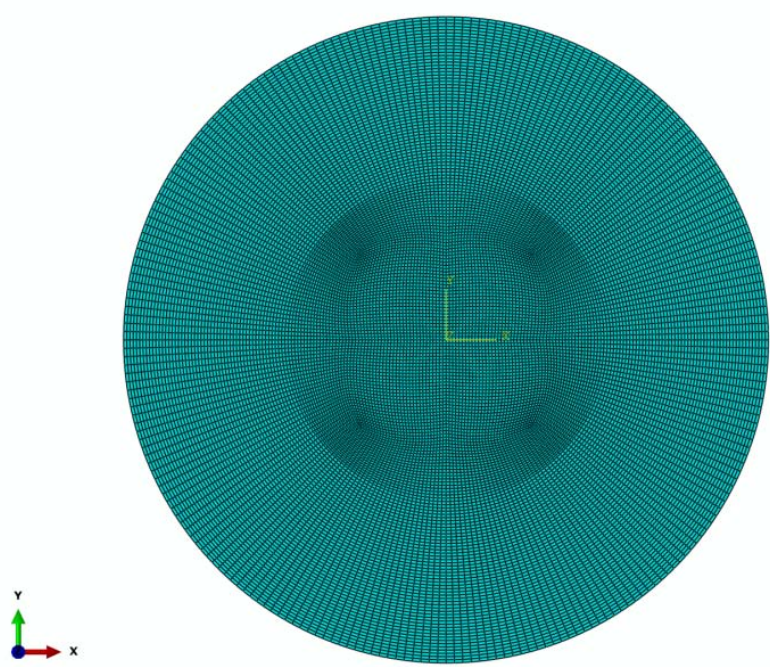

Elements: 20400, nodes: 20521

Figure 11. Meshes for a rectangular plate and solid cylinder with the finite element method. 

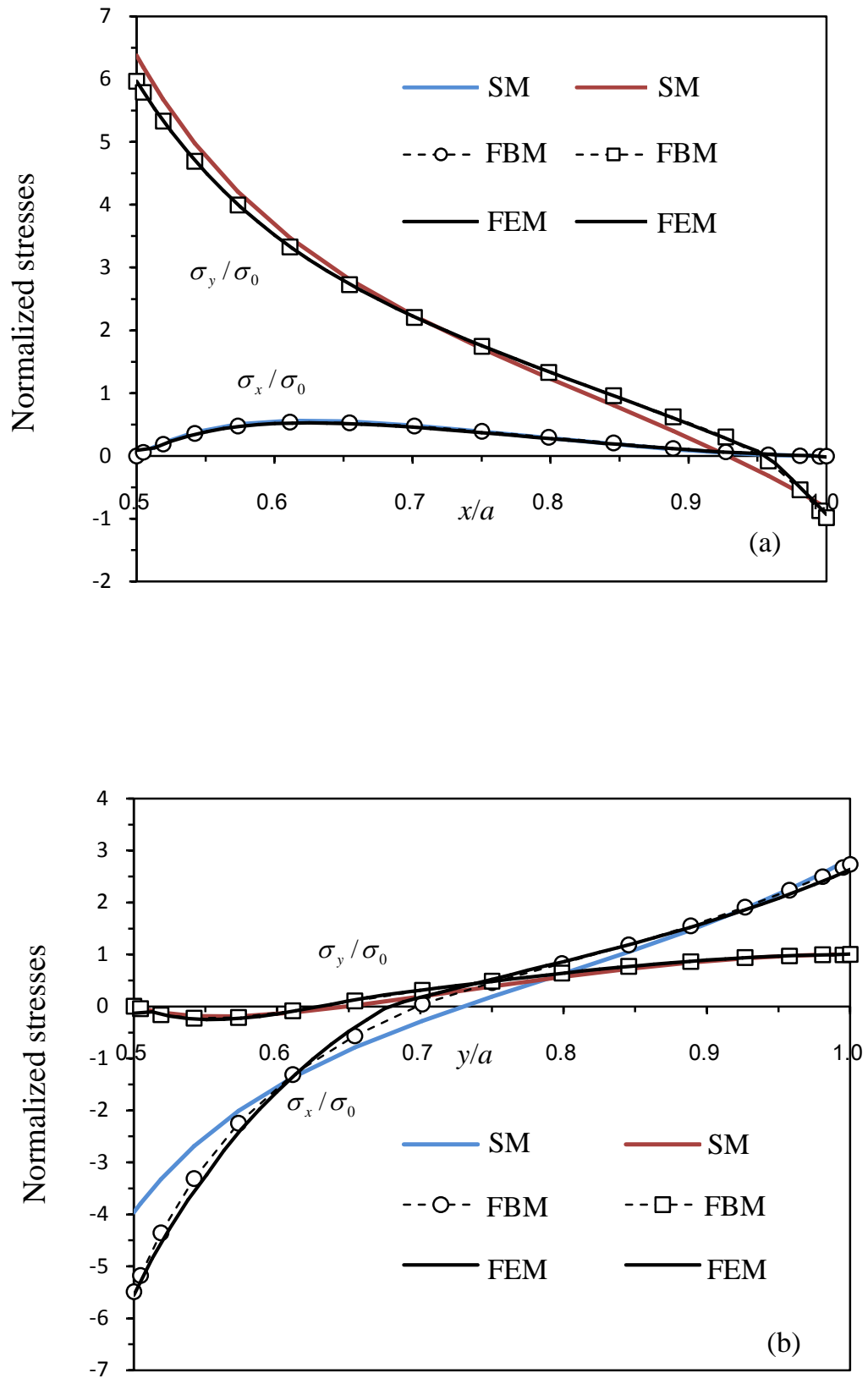

Figure 12. Normalized stress distributions when $\beta=0.5$ : (a) along the edge $\mathrm{AB}$; (b) along the edge CD. 

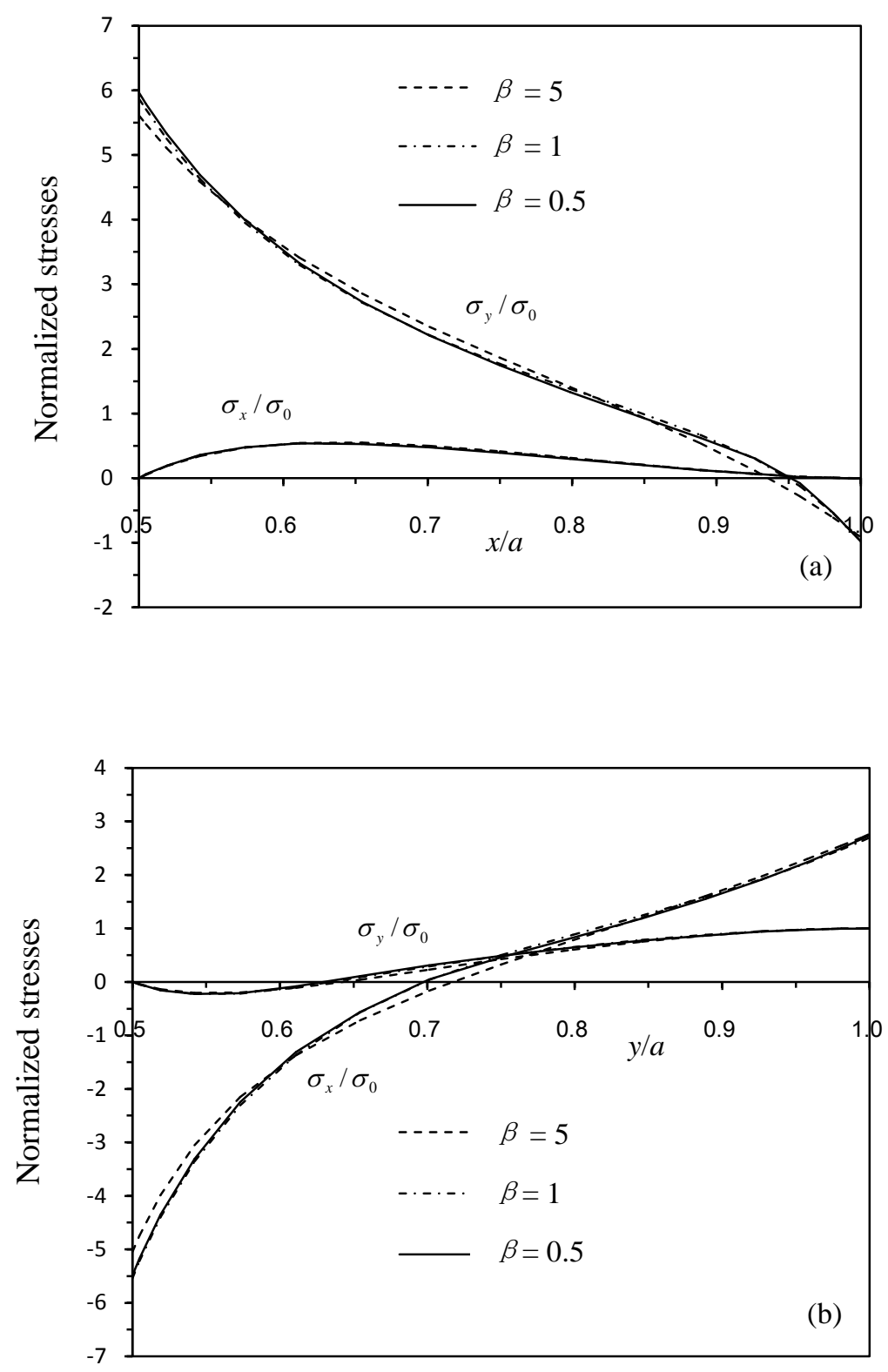

Figure 13. Normalized stress distributions for different characteristic factors: (a) along the edge AB; (b) along the edge CD.

\subsection{Hollow and solid cylindrical specimen under pressure load}

Finally, both a hollow and a solid cylinder are considered with a uniformly distributed pressure $\sigma_{0}$ on a partial outer surface as shown in Figure 13 with fracture and damage experimental specimen of asphalt-mixture materials in the National Laboratory of Changsha University of 
Science and Technology. Due to the symmetry of the problem, a quarter of the plate is modelled. Again, two blocks (I and II) are used and the interface is located at $\varphi(=\pi / 3)$ as shown in Figures 14(b)(c). For a hollow cylinder, we assume $r_{b}=2 r_{a}$ and for a solid cylinder $r_{b}=2$.

In this numerical modeling, the node numbers are selected as16 along two axes for each block and the smooth hyperbolic tangent model is adopted with characteristic factor $\beta=1,0.5$. Figures 15 and 16 show the normalized stress distributions $\sigma_{y} / \sigma_{0}$ and $\sigma_{x} / \sigma_{0}$ on edge $\mathrm{AB}$ and edge BC for the hollow and the solid cylinder respectively. The numerical solutions given by FEM [38] are shown on the same figures [38] for comparison. Seeing from these computational results, a reasonable accuracy was achieved by the meshless approach with continuous Young's modulus modeling to deal with nonlinear bimodular materials. It was noticed that the iteration becomes unstable when $\beta \leq 0.1$ and $m>5$. In addition, for the solid cylinder, the oscillation near the centre of cylinder is observed and this is caused by the inner radius selection, i.e. $r_{a} / r_{b}=0.01$ to model the solid cylinder. Therefore, in this case, the polar coordinate system for FBM should be considered.

\section{Conclusion}

The meshless finite block method was developed to study composites, rock-like and asphalt mixture material with different Young's moduli. As the constitute equation of stress and strain depends on the stress state in the domain, the partial differential equations are highly non-linear. It has been shown that the meshless FBM is one effective method to deal with bimodular material problems in engineering with high accuracy and convergence. The governing equations are formulated in a strong form and the efficiency of the method was demonstrated with three examples. The following conclusion can be made

(1) Linear and hyperbolic models are efficient for bimodular material analysis;

(2) Meshless approach is simple and accurate for nonlinear material property investigation;

(3) Characteristic parameter of material has slight influence in large selections.

Furthermore, this method can be extended straightaway to more complicated problems with bimodular materials such as elasto-plasticity, coupling with temperature and time dependent 
problems. In addition, the FBM method can be applied to a fracture problem where the stress singularity at crack tip is analytically determined.

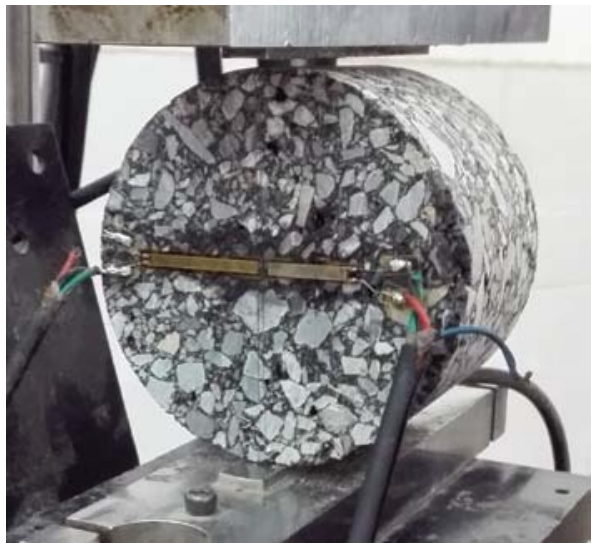

(a)

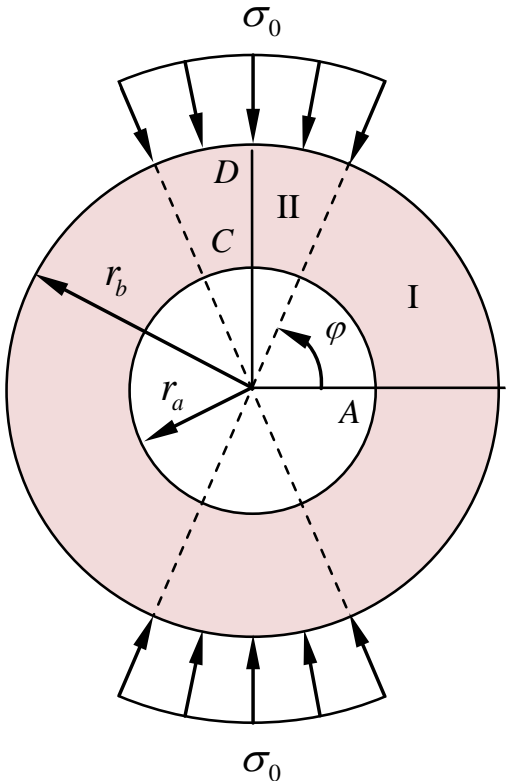

(b)

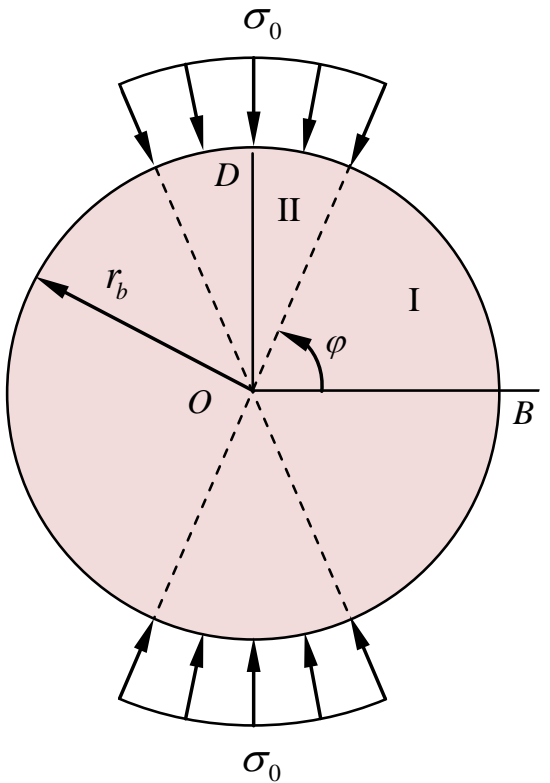

(c)

Figure 14. Geometry and boundary conditions of numerical modeling: (a) experiment of asphalt-mixture materials, (b) solid cylinder under compressive load and(c) hollow cylinder.

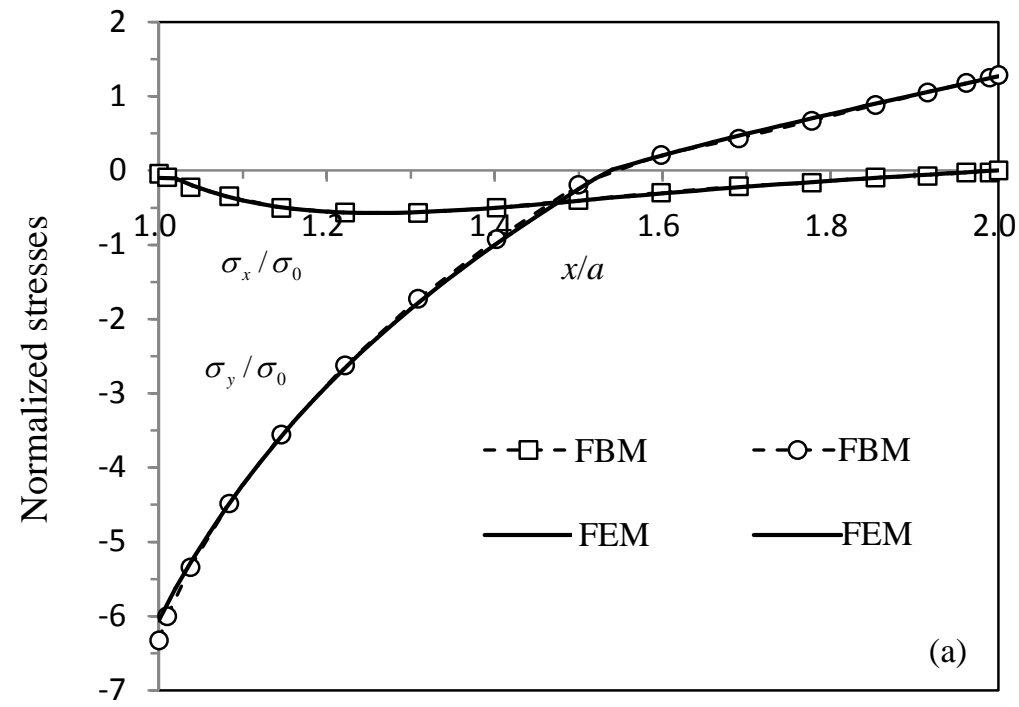




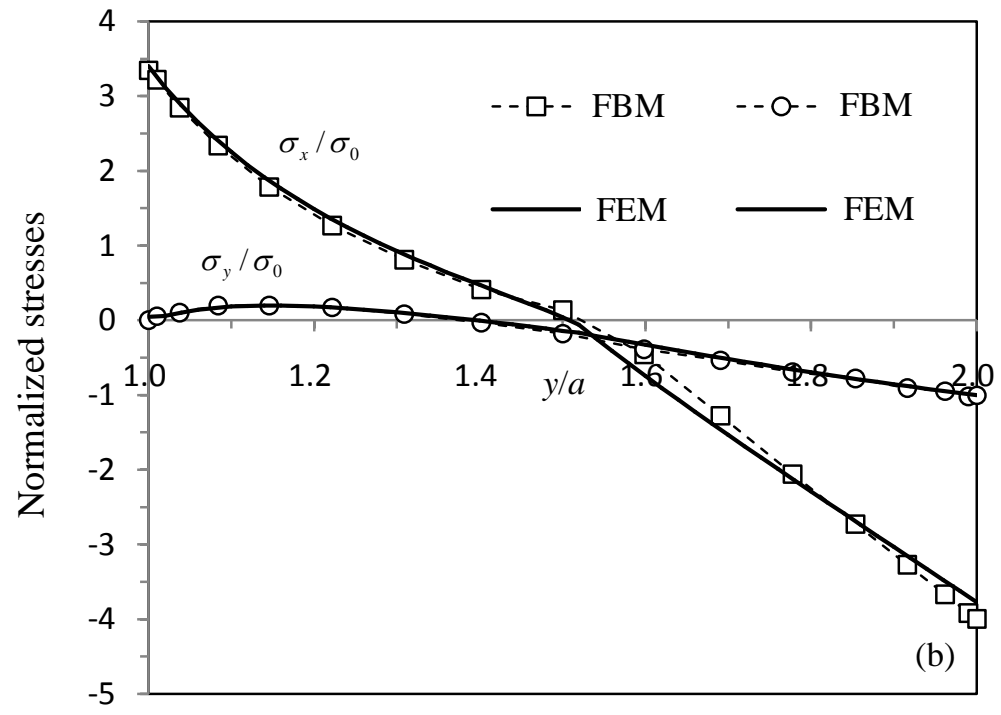

Figure 15. Normalized stress distributions when $\beta=0.5$ : (a) along the edge AB; (b) along the edge CD.

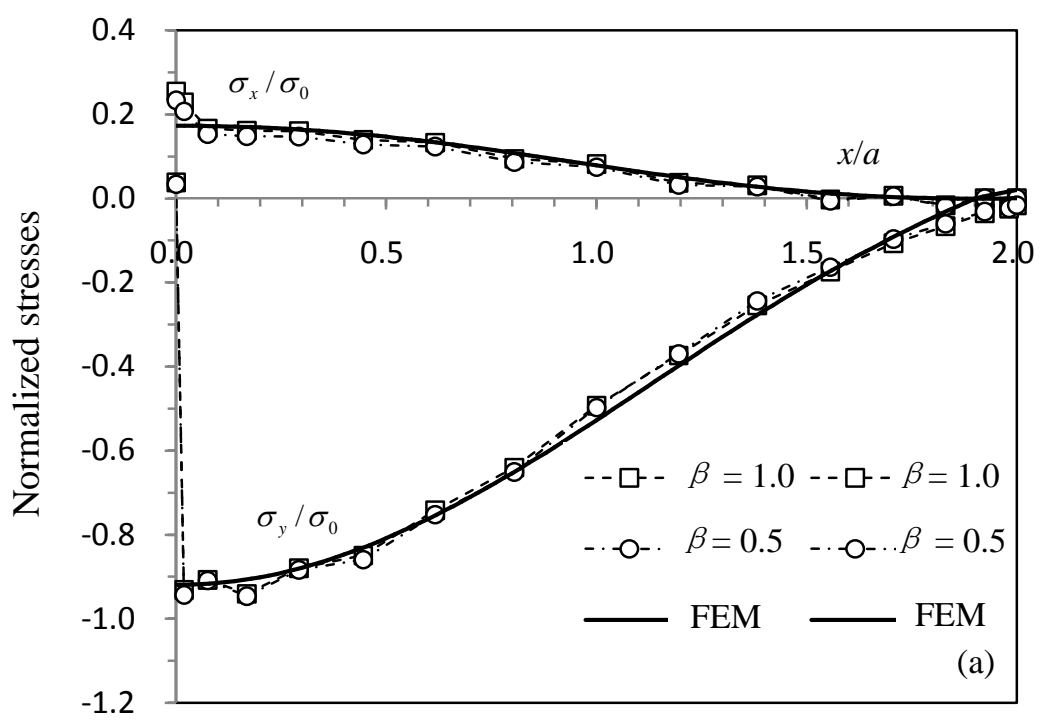




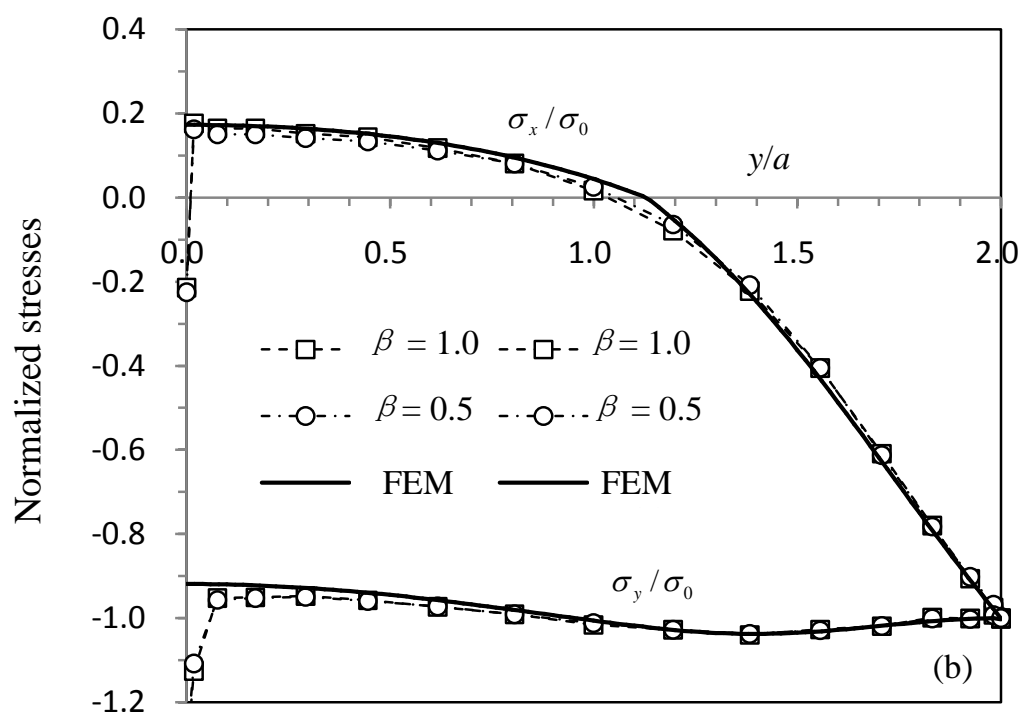

Figure 16. Normalized stress distributions for different characteristic factor $\beta$ : (a) along the edge AB; (b) along the edge CD.

\section{Acknowledgement}

The work was supported partially by the National Natural Science Foundation of China (No:51704040, 51608055) and the Construction Project of Science and Technology of Ministry of Transport of the People's Republic of China (No:2015318825120).

\section{References}

[1] Ambartsumyan, SA. The basic equations of the theory of elasticity for materials with different tensile and compressive strengths. Mekhanika Tverdogo Tela 1966; 2: 44-53.

[2] Jones RM. Stress-strain relations for materials with different moduli in tension and compression. AIAA Journal 1977; 15 (1):16-23.

[3] Medri G. A nonlinear elastic model for isotropic materials with different behavior in tension and compression. ASME Journal of Engineering Materials and Technology 1982;104 (1): 26-28. 
[4] Bert CW. Models for fibrous composites with different properties in tension and compression. ASME Journal of Engineering Materials and Technology 1977;99 (4): 344-349.

[5] Bert CW, Gordaninejad F. Transverse effects in bimodular composite laminates. Journal of Composite Materials 1983; 17 (4): 282-298.

[6] Reddy JN, Chao WC. Nonlinear bending of bimodular-material plates. International Journal of Solids and Structures 1983; 19 (3): 229-237.

[7] Srinivasan RS, Ramachandra LS. Large deflection analysis of bimodulus annular and circular plates using finite elements. Computers \& Structures 1989;31 (5): 681-691.

[8] Bruno D, Lato S, Sacco E. Nonlinear analysis of bimodular composite plates under compression. Computational Mechanics 1994;14 (1):28-37.

[9] Tseng YP, Jiang YC. Stress analysis of bimodulus laminates using hybrid stress plate elements. International Journal of Solids and Structures 1998; 35 (17): 2025-2028.

[10] Zinno R, Greco F. Damage evolution in bimodular laminated composites under cyclic loading. Composite Structures 2001;53 (4): 381-402.

[11] Ambartsumyan SA. Different Modulus Theory of Elasticity (in Russian) (Monograph), Moscow Science Publications, Physics and Mathematical Literature, 1982.

[12] Ambartsumyan SA. The Basic Equations and Relations of the Different-Modulus Theory of Elasticity of an Anisotropic Body. Mech. Solids 1969; 4(3): 48-56.

[13] Ambartsumyan SA, Khachatryan AA. The basic equations of the theory of elasticity for materials with different stiffness in tension and compression. Mech. Solids 1966; 1: 29-34.

[14] Ye ZM, Yu HR, Yao WJ. A new elasticity and finite element formulation for different Young modulus when tension and compression loadings. Journal of Shanghai University (English Edition) 2001;5(2): 89-92.

[15] Akhtar S, Khan S, Si Y. A three-dimensional finite element program for bimodular rocklike materials. Int. J. for Numerical and Analytical methods in Geomechanics 1988;12: 599-609. 
[16] Xie WH, Peng ZJ, Meng SH, Xu CH, Yi FJ, Du SY. WFMM model for bi-modulus orthotropic materials: Application to mechanical analysis of 4D-C/C composites. Composite Structures 2016; 150: 132-138.

[17] Yang H, Wang B. An analysis of longitudinal vibration of bimodular rod via smoothing function approach. Journal of Sound and Vibration 2008; 317:419-431.

[18] He X, Chen S, Sun J. Elasticity solution of simple beams with different modulus under uniformly distributed load. Eng. Mech. 2007;24:51-6.

[19] He X, Zheng Z, Chen S. Approximate elasticity solution of bending compression column with different tension-compression moduli. J Chongqing University 2008;3:023.

[20] Yao WJ, Ye ZM. Analytical solution of bending-compression column using different tension-compression modulus. Appl. Math. Mech. 2004;25:983-93.

[21] Gingold RA, Monaghan JJ. Smooth particle hydrodynamics: theory and application to non-spherical stars. Mon. Not. R. Astr. Soc. 1977;181:375-389.

[22] Lucy BL. A numerical approach to testing the fission hypothesis. Astron. J. 1977; 82(12):1013-1924.

[23] Nayroles B, Touzot G, Villon P. Generalizing the finite element method: diffuse approximation and diffuse elements. Computational Mechanics 1992;10: 307-318.

[24] Belytschko T, Lu YY, Gu L. Element-free Galerkin method. Int. J. Numerical Methods in Engineering 1994;37: 229-256.

[25] Liu WK, Jun S, Zhang Y. Reproducing kernel particle methods. Int. J. Numerical Methods in Engineering 1995;20: 1081-1106.

[26] Atluri SN, Zhu T. A new meshless local Peyrov-Galerkin (MLPG) approach to nonlinear problems in computational modelling and simulation. Comput. Model. Simul. Engng 1998; 3:187-196.

[27] Atluri SN, Zhu T. A new meshless local Peyrov-Galerkin (MLPG) approach in computational mechanics. Comput. Mech.1998; 22: 117-127.

[28] Atluri SN, Zhu T. The meshlesss local Peyrov-Galerkin (MLPG) approach for solving problems in elasto-statics. Comput. Mech. 1999;25:169-179. 
[29] Atluri SN, Shen S. The meshlesss local Peyrov-Galerkin (MLPG) method: a simple and less-costly alternative to the finite element and boundary element method. Comput. Model. Engng Sci. 2002;3: 11-52.

[30] Atluri SN. The Meshless Method (MLPG) for Domain and BIE Discretizations. Forsyth, GA, USA, Tech Science Press, 2004.

[31] Sladek J, Sladek V, Zhang Ch. Heat conduction analysis in nonhomogeneous anisotropic solid. Computational Mechanics, ZH Yao, MW Yuan, WX Zhong edited, Tsinghua University Press and Springer, 2004.

[32] Sladek J, Sladek V, Atluri SN. Meshless Local Petrov-Galerkin method for heat conduction problem in an anisotropic medium. Comput. Model. Engng Sci. 2004;6:309-318.

[33] Sladek V, Sladek J, Tanaka M, Zhang Ch. Local integral equation method for potential problems in functionally graded anisotropic materials. Engng Analy. with Boundary Elements 2005;29: 829-843.

[34] Wen PH, Cao P, Korakianitis T. Finite Block Method in elasticity. Engineering Analysis with Boundary Elements 2014; 46: 116-125.

[35] Li M, Wen PH. Finite block method for transient heat conduction analysis in functionally graded media. International Journal for Numerical Methods in Engineering 2014; 99(5): 372-390.

[36] Li M, Lei M, Munjiza A, Wen PH. Frictional contact analysis of functionally graded materials with Lagrange finite block method. International Journal for Numerical Methods in Engineering 2015; 103(6): 391-412.

[37] Li M, Meng LX, Hinneh P, Wen PH. Finite block method for interface cracks. Engineering Fracture Mechanics 2016; 156: 25-40.

[38] Pan QX. Research on Bimodulus Theory and its Application in Mechanics Analysis of Asphalt Pavement, PhD thesis, Changsha University of Science and Technology (in Chinese), 2018. 